\title{
Self-similar inverse semigroups and Smale spaces
}

\author{
Volodymyr Nekrashevych*
}

April 30, 2008

\author{
Kyiv Taras Shevchenko University \\ vul. Volodymyrska, 60 \\ Kyiv, 01033 \\ Ukraine \\ and \\ International University Bremen \\ P.O. Box 750561 \\ 28725 Bremen \\ Germany \\ E-mail: v.nekrashevych@iu-bremen.de
}

AMS Subject Classification: 20M18, 37D99, 37B05, 52C23.

\begin{abstract}
Self-similar inverse semigroups are defined using automata theory. Adjacency semigroups of $s$-resolved Markov partitions of Smale spaces are introduced. It is proved that a Smale space can be reconstructed from the adjacency semigroup of its Markov partition, using the notion of the limit solenoid of a contracting self-similar semigroup. The notions of the limit solenoid and a contracting semigroup is described.
\end{abstract}

\section{Introduction}

The aim of our paper is to show how self-similar inverse semigroups of automatic transformations appear naturally in dynamical system theory, in particular in connection with Smale spaces, minimal dynamical systems on Cantor sets and substitution tilings (the Penrose tilings, for instance).

We show, for example, that semigroups associated with tilings and Markov partitions of Smale spaces are generated by finite automata. This can be used to perform explicit computations and establishes an interesting connection between symbolic dynamics and semigroup theory. We also associate, in the spirit of the J. Kellendonk's construction, finitely generated semigroups (the adjacency semigroups) to Smale spaces of a special type. We show also that the Smale spaces are uniquely determined by the associated adjacency semigroups.

These results connect two different directions of investigation, which were developed independently before.

One direction investigates substitution tilings, Smale spaces and algebraic object related with them. Inverse semigroups were applied to the study of substitutional tilings by J. Kellendonk, who defined the tiling semigroups (see the articles [Kel97, Kel95, KL00] and [Pat99]). Inverse semigroups are essential in this situation, since substitutional tilings have typically a rich semigroup of partial symmetries, but are aperiodic, and thus have a small (trivial in the most cases) group of symmetries.

Interesting algebraic objects related with the Smale spaces are the Ruelle groupoids and the associated $C^{*}$-algebras, studied by I. F. Putnam and other authors (see [Put96, KPS97]). The

${ }^{*}$ The research was supported by Alexander von Humboldt Foundation and the Swiss National Science Foundation 
space of all (pointed) tilings, obtained from a substitution rule is a Smale space in a natural way [AP98, KP00].

The other direction, the theory of self-similar groups, was developed during the study of groups of automata for purely group-theoretical reasons. For the first definitions of a self-similar (stateclosed) group (or a group, generated by an automaton), see the works [Gri85, Sid98].

The class of self-similar groups contains many examples of groups with such interesting properties as intermediate growth, just-infiniteness, being an infinite finitely generated torsion group, etc. These examples have helped to solve many interesting problems in group theory. For examples of self-similar groups and their properties, see the papers [Gri80, Gri83, GS83, Gri98, BSV99, GLSŻ00]. See also the surveys [Gri00, GNS00, BGN03], and the book [Nek05] for a systematic study of self-similar groups and their relations to dynamical systems.

The study of self-similar groups had a dynamical flavor from the very beginning. Even the definitions of such groups where in a sense dynamical, since the groups where defined via their actions on graphs, topological spaces, or measure spaces.

The connections became more apparent during the subsequent investigations. For instance, there exist interesting applications of iterations of rational mappings to spectra of random walks on self-similar groups and their Schreier graphs (see [GŻ01, BG00]).

It was shown in [Nek05], that a naturally defined topological limit space $\mathcal{J}_{G}$ and a dynamical system s : $\mathcal{J}_{G} \longrightarrow \mathcal{J}_{G}$ on it is associated with every self-similar group $G$ belonging to an important class of contracting groups. Another object, associated to a contracting self-similar group, is the limit solenoid $\mathcal{S}_{G}$, which can be constructed as the inverse limit of the limit dynamical system $\left(\mathcal{J}_{G}, \mathrm{~s}\right)$. The limit solenoid has many properties, similar to the properties of the spaces of substitutional tilings. It is a union of leaves, where each leaf is a union of naturally defined tiles. The map s acts on the tiles as a substitution law (an inflation). In particular, the spaces of self-affine substitutional digit tilings (see definitions in [Vin00]) can be all obtained as limit solenoids of self-similar actions of the free abelian (or, in a more general setting, crystallographic) groups.

Self-similar groups can be naturally associated with branched self-coverings of topological spaces (for example, with the mappings of the complex sphere defined by rational functions) via the construction of the iterated monodromy group (see [Nek05, BGN03]). If the self-covering is expanding (if the rational function is sub-hyperbolic), then the iterated monodromy group $G$ is contracting and its limit space $\mathcal{J}_{G}$ is homeomorphic to the Julia set of the self-covering.

The notion of a self-similar inverse semigroup was introduced as a natural generalization of the notion of a self-similar group, see [Nek02, BGN03]. One of the first examples of a self-similar inverse semigroup was a variation of the tiling semigroup of the Penrose tiling (see its description in [BGN03] and in this article).

The current article is an attempt to generalize the mentioned results on limit spaces and iterated monodromy groups to the semigroup case.

The structure of the paper is the following. In Section 2 we define the notion of a self-similar inverse semigroup, its groupoid of germs and present some typical examples. Self-similar inverse semigroups act by local homeomorphisms on Markov shift spaces. We give the basic definitions of shift spaces in the first subsection of Section 2. The notion of self-similar inverse semigroup is defined in the second subsection. We use here the language of (non-deterministic) automata, similar to the group case. A previous definition given in [BGN03] did not use explicitly the notion of an automaton. The groupoid of germs is a natural object associated with every inverse semigroup of local homeomorphisms (see [BH99]) and is a convenient notion for self-similar semigroups. Selfsimilarity of the inverse semigroup can be, for example, formulated in terms of the groupoid of germs as the requirement that the shift induces an endomorphism of the groupoid.

Among the examples (Subsection 2.5) we present the self-similar inverse semigroups related to the "adic" transformations of the Cantor space defined by stationary Vershik-Bratelli diagrams and a variant of the tiling semigroup of the Penrose tiling.

The next section "Contracting self-similar semigroups" generalizes the notion of a contracting group and its limit solenoid to self-similar inverse semigroups. A self-similar inverse semigroup is called contracting if the iteration of the shift attracts every element of the groupoid of germs to a 
compact subset. We associate with every contracting self-similar inverse semigroup $H$ a metrizable finite-dimensional topological space $\mathcal{S}_{H}$ together with a homeomorphism s: $\mathcal{S}_{H} \longrightarrow \mathcal{S}_{H}$.

We apply in Section 4 the introduced notions and results to Smale spaces. Its first subsection contains the standard definitions of the Smale spaces (following [Rue78, Put96]) and the definition of an $s$-resolved Markov partition. A Markov partition is called $s$-resolved if every rectangle is a direct product (with respect to the canonical coordinates) of a clopen stable set and closed unstable set. In particular, if a Smale space has an $s$-resolved Markov partition, then its stable manifolds are 0-dimensional.

Subsection 4.2 contains the main results of our paper. We introduce a notion of the adjacency semigroup of an s-resolved Markov partition (Definition 4.2) and prove (Theorem 4.6) that it is self-similar contracting and that the limit dynamical system $\left(\mathcal{S}_{H}, \mathrm{~s}\right)$ of the adjacency semigroup $H$ is topologically conjugate with the Smale space. In particular, the action of the inverse semigroup on the shift space determines the Smale space uniquely.

Acknowledgments. The author is grateful to L. Bartholdi, T. Giordano, R. Grigorchuk, S. Margolis, B. Steinberg for useful discussions and interest and to the referee for many suggestions which helped to improve the paper.

\section{Self-similar inverse semigroups}

\subsection{Preliminary definitions}

Let $X,|X|>1$ be a finite set, called alphabet. We associate with every alphabet two spaces of sequences. One is the space $X^{\mathbb{N}}$ of infinite one-sided sequences of the form $x_{1} x_{2} \ldots$, the other is the space $X^{\mathbb{Z}}$ of bi-infinite sequences of the form $\ldots x_{-2} x_{-1} \cdot x_{0} x_{1} \ldots$, where the dot marks the place between the coordinates number -1 and number 0 . We introduce on the spaces $X^{\mathbb{N}}$ and $X^{\mathbb{Z}}$ the topology of the direct products of the discrete spaces $X$. Then both spaces are compact metrizable spaces without isolated points, i.e., homeomorphic to the Cantor space.

We denote for every subset $A \subseteq X^{\mathbb{Z}}$ and a finite sequence $u=a_{1} a_{2} \ldots a_{n}$ by $u A$ the set of sequences of the form $a_{1} a_{2} \ldots$ such that $a_{n+1} a_{n+2} \ldots \in A$.

We introduce also the following maps on the spaces $X^{\mathbb{N}}$ and $X^{\mathbb{Z}}$, called shifts:

$$
\begin{aligned}
\sigma\left(x_{1} x_{2} \ldots\right) & =x_{2} x_{3} \ldots \\
\sigma_{\mathbb{Z}}\left(\ldots x_{-2} x_{-1} \cdot x_{0} x_{1} \ldots\right) & =\ldots x_{-3} x_{-2} \cdot x_{-1} x_{0} \ldots
\end{aligned}
$$

The map $\sigma: X^{\mathbb{N}} \longrightarrow X^{\mathbb{N}}$ is an $|X|$-to-one continuous surjection and the map $\sigma_{\mathbb{Z}}: X^{\mathbb{Z}} \longrightarrow X^{\mathbb{Z}}$ is a homeomorphism. Note that $\sigma$ and $\sigma_{\mathbb{Z}}$ are shifts in different directions.

A subspace $\mathcal{F} \subseteq X^{\mathbb{N}}$ or $\mathcal{F}_{\mathbb{Z}} \subseteq X^{\mathbb{Z}}$ is called shift space (or just shift) if it is closed and shiftinvariant, i.e., if $\sigma(\mathcal{F}) \subseteq \mathcal{F}$, or $\sigma_{\mathbb{Z}}\left(\mathcal{F}_{\mathbb{Z}}\right)=\mathcal{F}_{\mathbb{Z}}$, respectively.

Important examples of shift spaces are the Markov shifts. Every Markov shift is defined by a set of admissible pairs $A \subseteq X \times X$. If $A$ is a set of admissible pairs, then the respective one-sided Markov shift $\mathcal{F}=\mathcal{F}(A)$ is defined as the set of all sequences $x_{1} x_{2} \ldots \in X^{\mathbb{N}}$ such that $\left(x_{n} ; x_{n+1}\right) \in A$ for all $n \geq 1$. Similarly, the two-sided Markov shift $\mathcal{F}_{\mathbb{Z}}=\mathcal{F}_{\mathbb{Z}}(A)$ is defined as the set of sequences $\left(\ldots x_{-2} x_{-1} \cdot x_{0} x_{1} \ldots\right) \in X^{\mathbb{Z}}$ such that $\left(x_{n} ; x_{n+1}\right) \in A$ for all $n \in \mathbb{Z}$.

If $\mathcal{F}$ is a one-sided Markov shift, then its natural extension is the two-sided Markov shift $\mathcal{F}_{\mathbb{Z}}$ defined by the same set of admissible pairs. For more on shift spaces, see the books [LM95, Kit98].

\subsection{Automata}

Definition 2.1. A (non-deterministic) automaton over an alphabet $X$ is a directed graph $\Gamma$ (loops and multiple edges are allowed) with the arrows labeled by pairs $(x ; y)$ of letters from $X$. The vertices of the graph are called the states of the automaton. 
We say that a transition $(u ; v)$, where $u, v \in X^{\mathbb{N}}$, is accepted by a state $q \in \Gamma$ if there is an infinite directed path starting in $q$ whose consecutive edges are labeled by the labels $\left(x_{1} ; y_{1}\right),\left(x_{2} ; y_{2}\right), \ldots$ so that $u=x_{1} x_{2} \ldots, v=y_{1} y_{2} \ldots$

If $\Gamma$ is an automaton, then its inverse is the automaton, obtained from $\Gamma$ by changing every label $(x ; y)$ to $(y ; x)$.

If a transition $(u ; v)$ is accepted by a state $q$ then we say also that the state $q$ defines the transition $(u ; v)$.

Our automata may be infinite (i.e., may have infinitely many states), but we always suppose that they are locally finite, i.e., that at every state only a finite number of arrows start.

If $\Gamma$ is an automaton and $q$ is its state, then a sequence $v \in X^{\mathbb{N}}$ is accepted by the state $q$ if there exists $u \in X^{\mathbb{N}}$ such that the transition $(v ; u)$ is accepted by $q$. The set of all sequences, accepted by $q$ is denoted $\operatorname{Dom} q$. It is easy to see that $\operatorname{Dom} q$ is a closed subset of the space $X^{\mathbb{N}}$.

The union $\operatorname{Dom} \Gamma=\bigcup_{q \in \Gamma} \operatorname{Dom} q$ is called the set of the sequences accepted by the automaton $\Gamma$. It is easy to see that the set Dom $\Gamma$ is invariant under the shift $\sigma$. If the automaton $\Gamma$ is finite then the set Dom $\Gamma$ is closed and is a shift space.

An automaton $\Gamma$ is said to be $\omega$-deterministic if for every states $q$ and for every $u \in X^{\mathbb{N}}$ there exists at most one path in $\Gamma$ starting in $q$ and labeled by $\left(x_{1} ; y_{1}\right),\left(x_{2} ; y_{2}\right), \ldots$, where $u=x_{1} x_{2} \ldots$. An $\omega$-deterministic automaton is said to be $\omega$-invertible if its inverse is $\omega$-deterministic.

If $\Gamma$ is an $\omega$-deterministic automaton and $q$ is its state, then the transformation defined by the state $q$ is the partial map, denoted $\Gamma_{q}$, with the domain $\operatorname{Dom} q$, mapping every sequence $u \in \operatorname{Dom} q$ to the unique sequence $v \in X^{\mathbb{N}}$ such that $(u ; v)$ is accepted by $q$.

Definition 2.2. Let $\Gamma$ be an automaton over the alphabet $X$ with the set of states $Q$. Then the dual automaton $\Gamma^{*}$ is the automaton over the alphabet $Q$ with the set of states $X$ such that we have an arrow from $x \in X$ to $y \in X$ labeled by a pair $(p ; q) \in Q^{2}$ if and only if there is an arrow from $p$ to $q$ labeled by the pair $(x ; y)$ in the automaton $\Gamma$.

It is easy to see that $\left(\Gamma^{*}\right)^{*}=\Gamma$. We need the notion of a dual automaton, since in many cases the dual automaton is easier to draw.

\subsection{Self-similar inverse semigroups}

Remark. We use left actions of semigroups, so that the image of a point $w$ under the action of a transformation $g$ is denoted $g(w)$ and in a product $g_{1} g_{2}$ the transformation $g_{2}$ acts first.

All inverse semigroups appearing in our article come together with a fixed action by partial permutation. Therefore, being absolutely accurate, one should actually change every instance of the term "inverse semigroup" in our article to the term "action of the inverse semigroup".

Definition 2.3. Let $H$ be an inverse semigroup acting by local homeomorphisms on a Markov shift $\mathcal{F} \subseteq X^{\mathbb{N}}$. We say that the action is self-similar if there exists an $\omega$-deterministic automaton $\Gamma(H)$ with the set of states $H$ such that $\operatorname{Dom} \Gamma(H)=\mathcal{F}$ and for every $g \in H$ the partial permutation $g$ coincides with the partial permutation $\Gamma_{g}$ defined by the state $g$ of the automaton $\Gamma(H)$.

Here by a local homeomorphism of a topological space we mean a homeomorphism between two open subsets. In our case these open sets have to be also closed, since the set of sequences accepted by a state is closed. Note that in conditions of the last definition the union of the domains of the elements of $H$ is the space $\mathcal{F}$ and the automaton $\Gamma(H)$ is $\omega$-invertible. When talking about an action of an inverse semigroup $H$ on a space $\mathcal{F}$, we always assume that the union of the domains of $H$ is the whole space $\mathcal{F}$.

We consider here only self-similar semigroups acting on a Markov space $\mathcal{F}$ by local homeomorphisms, though Definition 2.3 has sense in a more general situation (see, for example the paper [BGN03]).

The automaton $\Gamma(H)$ from Definition 2.3 is called the full automaton of the self-similar action.

We have the following easy criterion. (It shows also that the notion of a self-similar semigroup, adopted here coincides with the notion, introduced in [BGN03].) 
Proposition 2.1. An action of an inverse semigroup $H$ on a Markov shift space $\mathcal{F} \subseteq X^{\mathbb{N}}$ by local homeomorphisms is self-similar if and only if for every $g \in H$ the domain Dom $g$ is closed and for every $g \in H$ there exist a number $n \in \mathbb{N}$, a sequence $x_{1}, x_{2}, \ldots, x_{n} \in X$, a sequence $y_{1}, y_{2}, \ldots, y_{n} \in X$ and a sequence $h_{1}, h_{2}, \ldots, h_{n} \in H$ such that Dom $g=\bigsqcup_{i=1}^{n} x_{i} \operatorname{Dom} h_{i}$ and for every $w \in \operatorname{Dom} h_{i}$ we have $g\left(x_{i} w\right)=y_{i} h_{i}(w)$.

Here $\bigsqcup$ denotes disjoint union.

Proof. If the action is self-similar, then for every $g \in H$ let $e_{1}, e_{2}, \ldots, e_{n}$ be the set of all arrows of the automaton $\Gamma(H)$, starting in $g$. If $h_{i}$ is the end of the arrow $e_{i}$ and the arrow is labeled by $\left(x_{i} ; y_{i}\right)$, then the sequences $\left(x_{1}, x_{2}, \ldots, x_{n}\right),\left(y_{1}, y_{2}, \ldots, y_{n}\right)$ and $\left(h_{1}, h_{2}, \ldots, h_{n}\right)$ satisfy the condition of the proposition. The sets $x_{i}$ Dom $h_{i}$ are disjoint by the definition of an $\omega$-deterministic automaton.

On the other hand, if for every $g \in H$ such sequences $\left(x_{1}, x_{2}, \ldots, x_{n}\right),\left(y_{1}, y_{2}, \ldots, y_{n}\right)$ and $\left(h_{1}, h_{2}, \ldots, h_{n}\right)$ exist, then we can construct the automaton $\Gamma(H)$. We just have to draw $n$ arrows $e_{i}$ from $g$ to $h_{i}$ labeled by $\left(x_{i} ; y_{i}\right)$. Let us prove that then the state $g$ of the constructed automaton $\Gamma(H)$ defines the transformation $g$.

If $a_{1} a_{2} \ldots \in \mathcal{F}$ is a sequence belonging to the domain of $g$, then the sequence $a_{2} a_{3} \ldots$ belongs to the domain of the a transformation $f_{1}=h_{i}$, where $a_{1}=x_{i}$ and $g\left(a_{1} a_{2} \ldots\right)=y_{i} f_{1}\left(a_{2} a_{3} \ldots\right)$. Let us denote $y_{i}=b_{1}$. Repeating the same arguments for $a_{2} a_{3} \ldots$, then for $a_{3} a_{4} \ldots$, etc., we see that if $b_{1} b_{2} \ldots=g\left(a_{1} a_{2} \ldots\right)$ then there exists a sequence $f_{0}=g, f_{1}, f_{2}, \ldots$ such that the vertex $f_{k}$ is connected by an arrow labeled by $\left(a_{k+1} ; b_{k+1}\right)$ with the vertex $f_{k+1}$ for all $k \geq 0$. This gives us the necessary path, showing that the state $g$ of the automaton $\Gamma(H)$ admits the transition $\left(a_{1} a_{2} \ldots ; g\left(a_{1} a_{2} \ldots\right)\right)$.

On the other hand, suppose that the state $g$ admits the transition $\left(a_{1} a_{2} \ldots ; b_{1} b_{2} \ldots\right)$. Then there exists a path in the automaton $\Gamma(H)$ starting in $g$, labeled by the consecutive labels $\left(a_{k} ; b_{k}\right)$, $k=1,2, \ldots$, and passing through the consecutive vertices $g=f_{0}, f_{1}, \ldots$ Then we have, by construction of the automaton $\Gamma(H)$, that $a_{1} a_{2} \ldots a_{k} \operatorname{Dom} f_{k} \subset \operatorname{Dom} g$ and

$$
g\left(a_{1} a_{2} \ldots a_{k} x_{k+1} x_{k+2} \ldots\right)=b_{1} b_{2} \ldots b_{k} f_{k}\left(x_{k+1} x_{k+2} \ldots\right)
$$

for all $k \geq 1$ and $x_{k+1} x_{k+2} \ldots \in \operatorname{Dom} f_{k}$. But

$$
\operatorname{Dom} g \supset \bigcap_{k \geq 1} a_{1} a_{2} \ldots a_{k} \operatorname{Dom} f_{k}=\left\{a_{1} a_{2} a_{3} \ldots\right\},
$$

since the sets $a_{1} a_{2} \ldots a_{k}$ Dom $f_{k}$ are compact and form a descending sequence, so that the intersection is not empty. Similarly,

$$
\bigcap_{k \geq 1} b_{1} b_{2} \ldots b_{k} \operatorname{Ran} f_{k}=\left\{b_{1} b_{2} b_{3} \ldots\right\}
$$

and consequently, $g\left(a_{1} a_{2} \ldots\right)=b_{1} b_{2} \ldots$

Let us show that the constructed automaton is $\omega$-deterministic. Let $a_{1} a_{2} \ldots \in X^{\mathbb{N}}$ and $g \in$ $H$ be arbitrary. Then it follows from the previous consideration that $a_{1} a_{2} \ldots \in$ Dom $g$ if and only if there exists a path $e_{1} e_{2} \ldots$ in the automaton, starting in the state $g$ and labeled by $\left(a_{1} ; b_{1}\right),\left(a_{2} ; b_{2}\right), \ldots$ It also follows that the first arrow $e_{1}$ is necessary the arrow corresponding to the unique set $a_{1}$ Dom $h$ in the decomposition Dom $g=\bigsqcup x_{i}$ Dom $h_{i}$ to which belongs the sequence $a_{1} a_{2} \ldots$. Hence the first arrow $e_{1}$ and similarly all the subsequent arrows $e_{i}$ of the path $e_{1} e_{2} \ldots$ are uniquely determined by the sequence $a_{1} a_{1} \ldots$

The full automaton $\Gamma(H)$ is infinite if the semigroup $H$ is infinite. On the other hand, finite automata generate self-similar inverse semigroups in the following sense.

Definition 2.4. If $\Gamma$ is a finite $\omega$-invertible automaton, then the inverse semigroup generated by $\Gamma$ is the inverse semigroup acting on the space Dom $\Gamma$ generated by the partial permutations of the form $\Gamma_{q}$, where $q$ runs through the set of all states of the automaton $\Gamma$. 
The following proposition is an easy corollary of Proposition 2.1.

Proposition 2.2. An inverse semigroup generated by a finite automaton is self-similar (if it acts on Dom $\Gamma$ by local homeomorphisms).

\subsection{Groupoid of $H$-germs}

An important notion related to self-similar inverse semigroups is the associated groupoid. Recall that groupoid is a small category of isomorphisms (see a more explicit definition in [Pat99, Ren80]).

Let $H$ be a self-similar inverse semigroup acting on a Markov space $\mathcal{F}$. The groupoid of $H$-germs (denoted $\mathcal{G}(H)$ ) is the set of the equivalence classes of pairs $(h, w)$, where $h \in H$ and $w \in \operatorname{Dom} h$. Two pairs $\left(h_{1}, w_{1}\right)$ and $\left(h_{2}, w_{2}\right)$ are considered to be equivalent if and only if $w_{1}=w_{2}$, $h_{1}\left(w_{1}\right)=h_{2}\left(w_{2}\right)$ and the respective paths in the full automaton $\Gamma(H)$ starting in $h_{1}$ and $h_{2}$ and defining the transitions $\left(w_{1} ; h\left(w_{1}\right)\right)=\left(w_{2} ; h_{2}\left(w_{2}\right)\right)$ are cofinal, i.e., there exists a number $n_{0}$ such that the edge number $n$ of the first path coincides with the edge number $n$ of the second path for every $n>n_{0}$.

We will identify the germs with the respective pairs $(h, w)$ and with the paths in $\mathcal{G}(H)$ starting in $h$ and defining the transition $(w ; h(w))$.

We define the multiplication of the elements of the groupoid $\mathcal{G}(H)$ by the rules

$$
\left(h_{1}, w_{1}\right) \cdot\left(h_{2}, w_{2}\right)=\left(h_{1} h_{2}, w_{2}\right),
$$

where the product is defined if and only if $h_{2}\left(w_{2}\right)=w_{1}$. Then the inverse to the pair $(h, w)$ is the pair $\left(h^{-1}, h(w)\right)$.

It is easy to see that the associated groupoid $\mathcal{G}(H)$ is well defined. Our construction is a particular case of a general notion of the groupoid of germs of an inverse semigroup of local homeomorphisms. The groupoid of germs comes with a naturally defined germ topology (see [BH99]) and is an étale ( $r$-discrete) locally compact groupoid. Namely, a neighborhood of a germ $(h, w)$ is determined by a number $n \in \mathbb{N}$ and consists of all the germs $\left(h, w^{\prime}\right)$ such that the corresponding paths in $\Gamma(H)$ have a common beginning of length at least $n$.

The shift $\sigma: \mathcal{F} \rightarrow \mathcal{F}$ induces an endomorphism (functor) $\sigma_{*}: \mathcal{G}(H) \longrightarrow \mathcal{G}(H)$ defined by the map deleting the first edge of a path in the full automaton $\Gamma(H)$. It is easy to see that the shift

$\sigma_{*}$ agrees with the cofinality relation, thus is a well defined map of the groupoid. It follows also directly from the definition that $\sigma_{*}$ is an étale functor.

Definition 2.5. A self-similar inverse semigroup is said to be recurrent if the shift endomorphism $\sigma_{*}$ of the associated groupoid is onto.

Note that if the inverse semigroup acting on a space $\mathcal{F}$ is recurrent then $\sigma(\mathcal{F})=\mathcal{F}$, since the space $\mathcal{F}$ is identified with the space of units (with the set of objects) of the groupoid $\mathcal{G}(H)$.

\section{$2.5 \quad$ Examples}

Self-similar groups. A group $G$ acting by permutations on the space $X^{\mathbb{N}}$ is said to be selfsimilar (see [Nek05]) if for every $g \in G$ and every $x \in X$ there exist $h \in G$ and $y \in X$ such that $g(x w)=y h(w)$ for every $w \in X^{\mathbb{N}}$.

There exist many interesting examples of self-similar group actions. For example, many branch groups [Gri00] are self-similar. Branch groups often have interesting properties, rare among other classes of groups (many of them are just-infinite, have intermediate growth, are infinite torsion groups, etc.). An example of a self-similar branch group is the Grigorchuk group (see [Gri80, Har00]).

Another class of self-similar groups are the iterated monodromy groups of (branched) selfcoverings of topological spaces (for example, of rational functions on the complex sphere). For the definition of the iterated monodromy groups and their basic properties see [Nek05] and [BGN03]. 
The iterated monodromy group of a self-covering map $f$ contains much of the information about the dynamics of the map. In particular, in many cases the Julia set of $f$ can be reconstructed from its iterated monodromy group.

Let us give one of important examples of self-similar group actions. It is the action of the group $\mathbb{Z}$ on the space $\{0,1\}^{\mathbb{N}}$ generated by the transformation $a$, called the adding machine and defined by the rules:

$$
a(0 w)=1 w, \quad a(1 w)=0 a(w),
$$

where $w \in\{0,1\}^{\mathbb{N}}$ is arbitrary. It is easy to see that the transformation $a$ acts on the sequences from $\{0,1\}^{\mathbb{N}}$ in the same way as addition of 1 to a dyadic integer. More precisely, $a\left(x_{0} x_{1} \ldots\right)=y_{0} y_{1} \ldots$ is equivalent to the equality

$$
1+\sum_{k=0}^{\infty} x_{k} \cdot 2^{k}=\sum_{k=0}^{\infty} y_{k} \cdot 2^{k}
$$

in the ring $\mathbb{Z}_{2}$ of dyadics.

The adding machine action of $\mathbb{Z}$ is generated by the automaton shown on the left-hand side of Figure 1. On the right-hand side of the Figure the dual automaton is shown.

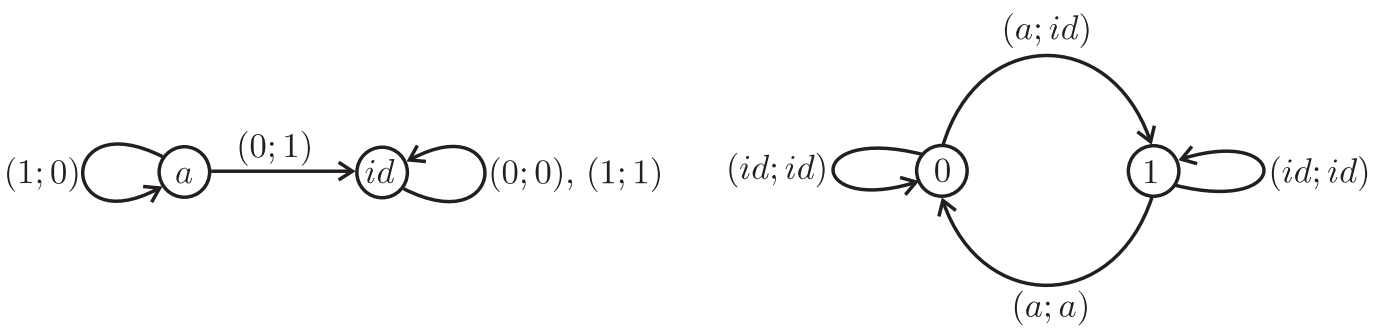

Figure 1: Adding machine.

"Adic" transformations. An interesting class of transformations defined by finite automata are "adic" transformations, introduced by Vershik.

Recall that a Bratelli diagram $\mathcal{D}$ is defined by a sequence of finite sets $V_{0}, V_{1}, \ldots$ (sets of the vertices of the diagram) and a sequence of finite sets $E_{1}, E_{2}, \ldots$ (sets of the edges of the diagram), together with maps $s_{i}: E_{i} \longrightarrow V_{i-1}$ and $r_{i}: E_{i} \longrightarrow V_{i}$. If $e \in E_{i}$ is an edge, then the vertex $s_{i}(e) \in V_{i-1}$ is the source and the vertex $r_{i}(e) \in V_{i}$ is the range of $e$.

A Vershik-Bratelli diagram is a Bratelli diagram with partial orders on every set $E_{i}$. Two edges must be comparable in this order if and only if they have equal ranges.

If we have a Vershik-Bratelli diagram $\mathcal{D}$, then the order on the edges induces the lexicographical order on the paths of $\mathcal{D}$. Namely a (finite or infinite) path $\left(e_{1}, e_{2}, \ldots\right)$ (i.e., such a sequence $e_{i} \in E_{i}$ that $\left.r_{i}\left(e_{i}\right)=s_{i+1}\left(e_{i+1}\right)\right)$ is less than a path of the same length $\left(f_{1}, f_{2}, \ldots\right)$ if and only if for some index $i_{0}$ we have that $e_{i}=f_{i}$ for all $i>i_{0}$ and the edge $e_{i_{0}}$ is less than $f_{i_{0}}$. It is easy to prove that for every infinite path $w$, which is not maximal in the lexicographical order there exists exactly one infinite path $\tau(w)$ (the next path to $w$ ) minimal among the paths which are greater than $w$.

The transformation $\tau$ defined on the set of non-maximal paths is called the adic transformation, defined by the Vershik-Bratelli diagram.

If the maximal path $w_{1}$ is unique and the minimal path $w_{0}$ is unique, then one can define $\tau\left(w_{1}\right)=w_{0}$ and the transformation $\tau$ will be a minimal homeomorphism of the space of infinite paths in the diagram $\mathcal{D}$. In fact, every minimal homeomorphism of the Cantor space is of this form, i.e., is conjugate to an adic transformation (see [GPS95]).

A stationary Vershik-Bratelli diagram $\mathcal{D}$ is a Vershik-Bratelli diagram defined by constant sequences $V_{i}=V, E_{i}=E$, and $s_{i}=s: E \longrightarrow V, r_{i}=r: E \longrightarrow V$ such that all the orderings on $E_{i}=E$ are equal. The space of all infinite paths in a stationary diagram is obviously a Markov shift over the alphabet $X=E$. 
For every $n \in \mathbb{N}$ the $n$th telescoping $\mathcal{D}^{(n)}$ of a stationary Vershik-Bratelli diagram $\mathcal{D}$ is the diagram defined by the same set of vertices $V$, by the set of edges $E^{(n)}$ equal to the set of paths $e=\left(e_{1}, e_{2}, \ldots, e_{n}\right)$ of length $n$ in the original diagram, the maps $s^{(n)}(e)=s\left(e_{1}\right), r^{(n)}(e)=r\left(e_{n}\right)$ and the lexicographic order on $E^{(n)}$ induced by the order on $E$. The telescoping changes the adic transformation to a conjugate one. See more on Vershik-Bratelli diagrams and adic transformations in the papers [HPS92, GPS95].

Suppose that we have a stationary diagram $\mathcal{D}$, which has a unique minimal and a unique maximal path. For every edge $a$ of the first level of the diagram, denote by $\tau_{a}$ the restriction of the minimal homeomorphism $\tau$ onto the set of paths with the first edge $a$. Denote by $H_{\mathcal{D}}$ the inverse semigroup generated by the transformations $\tau_{a}, a \in E$. We have the following result (see [Nek02]).

Theorem 2.3. Let $\mathcal{D}$ be a stationary Vershik-Bratelli diagram with a single maximal and a single minimal path. Then there exists $n \in \mathbb{N}$ such that the semigroup $H_{\mathcal{D}^{(n)}}$ is self-similar.

For example, consider the stationary Vershik-Bratelli diagram shown on the upper part of Figure 2. We introduce the ordering of the edges as follows:

$$
a_{0}<a_{1}<a_{2}, \quad b_{0}<b_{1} .
$$

Then the adic transformations $\tau_{x}$ are defined by the dual automaton, shown on the lower part of Figure 2. Here a label $\left(a ; b_{1}, b_{2}, \ldots, b_{n}\right)$ means that we have at this place $n$ arrows with the labels $\left(a ; b_{i}\right)$. The transformations $1_{x}$ are the identical transformations, with the domain equal to the set of paths with the first edge $x$. We have omitted the loops, defining the identical transformations $1_{x}$, since it is obvious how to draw them (one must only not forget that some of transitions $\left(1_{x} ; 1_{y}\right)$ are prohibited by the Vershik-Bratelli diagram).
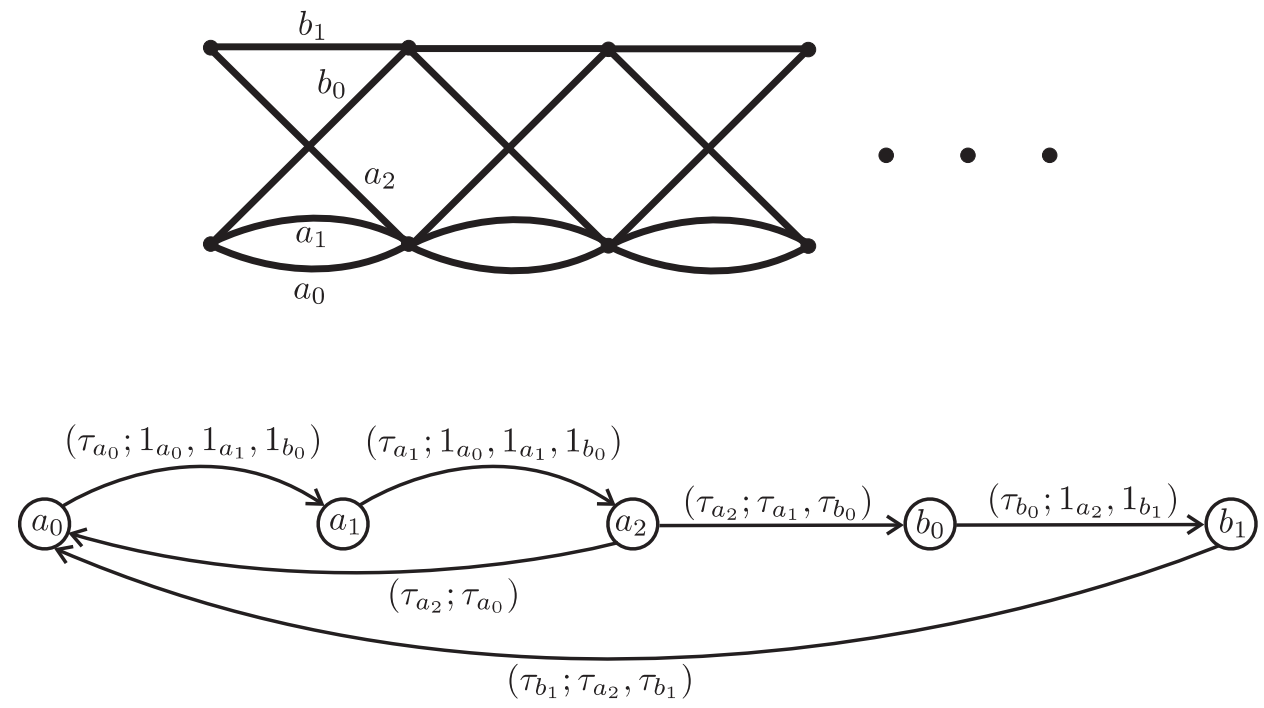

Figure 2: Adic transformations.

Another example of a homeomorphism defined by a stationary Vershik-Bratelli diagram is the binary adding machine described before. It is defined by the set $V$ consisting of a single point and the set $E=\{0,1\}$ with the ordering $0<1$.

Penrose tiling. The following example of a self-similar semigroup is defined in [BGN03]. It is an example similar to the tiling semigroup of J. Kellendonk, different from it only in some technical details. 
Recall, that a Penrose tiling (see [Pen84, Gar77, GS87]) is a tiling of the plane by the triangles of two types shown on Figure 3, which has to satisfy the matching rules.

The angles of the triangles are equal to $\frac{\pi}{5}, \frac{2 \pi}{5}, \frac{2 \pi}{5}$ and to $\frac{3 \pi}{5}, \frac{\pi}{5}, \frac{\pi}{5}$. They can be cut from a regular pentagon by the diagonals issued from a common vertex.

The matching rules require that the common vertices of two tiles are marked by the same color (black or white) and that the arrows on the adjacent sides of the triangles point at the same direction (see Figure 3 for the marking of the vertices and the arrows).
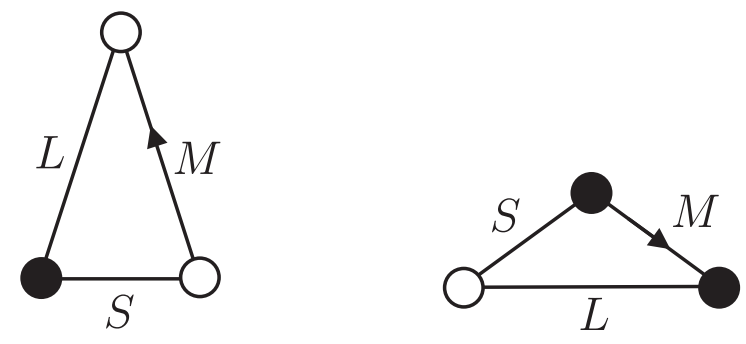

Figure 3: Tiles and matching rules.

It follows from the matching rules that if we mark the sides of the tiles by the letters $S, L$ and $M$ in the way it is done on Figure 3, then in every Penrose tiling the common sides of any two adjacent tiles will be marked by the same letter.

One can check, just considering all possible patches of Penrose tilings of sufficient size, that the tiles of any Penrose tiling can be grouped into blocks of two or three tiles, as it is shown on Figure 4 (the mirror images are also allowed).
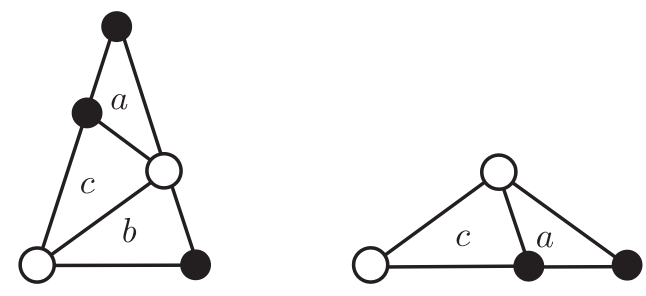

Figure 4: Inflation

The grouping into blocks is unique [GS87] and it is easy to see that the blocks are similar to the original tiles and that the blocks also form a Penrose tiling. This tiling is called the inflation of the original one.

The inflation procedure defines an encoding of the tiles of Penrose tilings by infinite sequences from the Markov shift $\mathcal{F}$ over the alphabet $X=\{a, b, c\}$ defined by the set of admissible pairs $A=X \times X \backslash\{(b ; a)\}$. Namely, let $T$ be a tile of a Penrose tiling. Then $T$ is a subset of a tile $T_{n}$ of the Penrose tiling obtained by the $n$th iteration of the inflation procedure. Let $a_{n} \in X$ be the label of the tile $T_{n}$ on Figure 4, according to its participation in the inflation. Then $a_{0} a_{1} \ldots \in \mathcal{F}$ is the label of $T$.

This encoding is one-to-one, except for the case when a tiling has a non-trivial symmetry group (this group is always finite and isomorphic either to $\mathbb{Z} / 2 \mathbb{Z}$ or to the dihedral group $\mathbb{D}_{5}$ of order 10). If the tiling has a non-trivial symmetry group, then the tiles belonging to one orbit of the symmetry group correspond to one sequence (see analysis of this situation in [BGN03]). 
Define transformations $L, M, S$ of the space $\mathcal{F}$ by the formulae:

$$
\begin{array}{rlrl}
S(a w) & =c w & L(a a w) & =b \cdot S(a w) \\
S(b w) & =b \cdot M(w) & L(a b w) & =a \cdot M(b w) \\
S(c w) & =a w & L(a c w) & =a \cdot M(c w) \\
& & L(b b w) & =b \cdot S(b w) \\
M(a w) & =a \cdot L(w) & L(b c w) & =a \cdot S(c w) \\
M(b w) & =c w & L(c w) & =c \cdot L(w) \\
M(c a w) & =c \cdot M(a w) & & \\
M(c b w) & =b b w & & \\
M(c c w) & =b c w
\end{array}
$$

If $w \in \mathcal{F}$ corresponds to a tile $T$ of the Penrose tiling, then $S(w), M(w)$ and $L(w)$ correspond to the tiles having a common side with $T$, marked on Figure 3 by the letters $S, M$ and $L$ respectively. This is also proved just by considering all possible patches of sufficient size in the Penrose tilings (actually, by considering one sufficiently large patch, since all Penrose tilings are locally isomorphic).

If we denote by $S_{x}, M_{x}, L_{x}$ the restrictions of $S, M$ and $L$ onto the sets of sequences starting with $x$, for $x \in\{a, b, c\}$, then the transformations $S_{x}, M_{x}, L_{x}, x \in\{a, b, c\}$ are defined by the dual automaton shown on Figure 5. We have not written the loops defining the idempotents $1_{x}$.

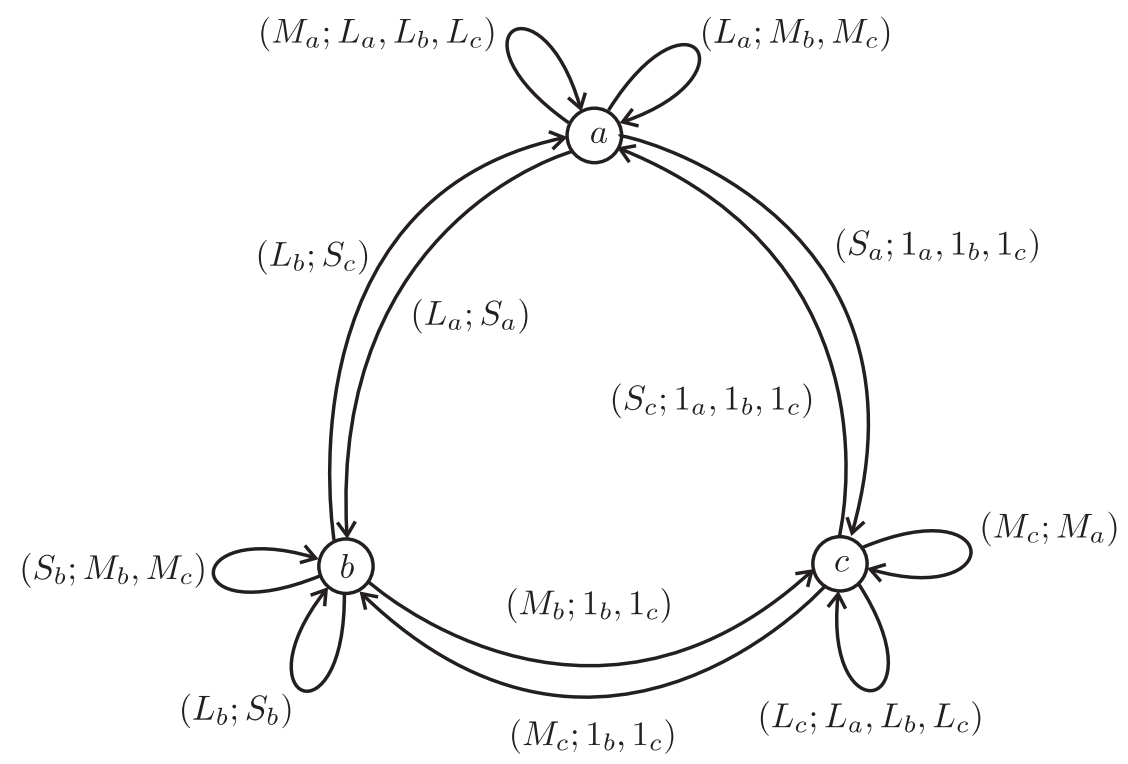

Figure 5: Adjacency semigroup of the Penrose tilings.

\section{Contracting self-similar semigroups}

\subsection{Definitions}

Definition 3.1. A subautomaton of an automaton $\Gamma$ is a subgraph $\Gamma_{1}$ of $\Gamma$ such that every arrow of $\Gamma$ starting in a vertex of $\Gamma_{1}$ also belongs to $\Gamma_{1}$.

So, a sub-automaton $\Gamma_{1}$ is uniquely determined by the corresponding subset of states of $\Gamma$. If $\Gamma$ is $\omega$-deterministic and $\Gamma_{1}$ is a sub-automaton of $\Gamma$, then every state $q$ of $\Gamma_{1}$ defines the same transformation as the state $q$ of $\Gamma$. 
Definition 3.2. Let $H$ be a self-similar semigroup and let $\Gamma(H)$ be its full automaton. We say that the semigroup $H$ is contracting if there exists a finite subautomaton $\mathcal{N} \subset \Gamma(H)$ such that every infinite path $e_{1}, e_{2}, \ldots$ in the automaton $\Gamma(H)$ has only a finite number of arrows $e_{i}$ outside $\mathcal{N}$.

The minimal set $\mathcal{N}$ satisfying the conditions of the definition is called the nucleus of the action.

Proposition 3.1. The nucleus exists if the semigroup is contracting. It consists of all the states $g$ of $\Gamma(H)$ such that there exists a directed path to $g$ from a directed cycle in $\Gamma(H)$.

Proof. Suppose that the semigroup is contracting. Let $N$ be any finite subautomaton satisfying the conditions of Definition 3.2. Every cycle of $\Gamma(H)$ must belong to $N$, since there exists a path going infinitely many times through each of its arrows. Consequently, every end of a path starting in a vertex of a cycle must belong to $N$.

Let us prove that the set $C$ of the states $g$ which are ends of the directed paths starting in elements of the cycles is the nucleus. We have shown that it is a sub-automaton of any finite automaton, satisfying the conditions of Definition 3.2. It remains to show that $C$ itself satisfies the conditions of Definition 3.2.

Let $e_{1}, e_{2}, \ldots$ be an arbitrary infinite directed path. Its all but finite number of arrows belong to a finite set satisfying the conditions of Definition 3.2. Hence, some arrow $e_{i}$ appears in this path twice. All the states between these two appearances belong to a cycle in $\Gamma(H)$. Consequently, all the states appearing after them belong to $C$.

Lemma 3.2. Let $H$ be a contracting self-similar inverse semigroup acting on a Markov shift $\mathcal{F}$. Then for every $g \in H$ there exists $n_{0} \in \mathbb{N}$ such that every path in $\Gamma(H)$ of length $\geq n_{0}$ which starts in $g$ ends in an element of the nucleus.

Proof. Let $w \in \operatorname{Dom} g$ be an arbitrary sequence. Then the path starting in $g$ and defining the transition $(w ; g(w))$ has all edges inside the nucleus $\mathcal{N}$, except for some finite beginning. Let $n_{w}$ be the length of this beginning, and let $h_{w} \in \mathcal{N}$ be its end. Then we have for every $u \in \operatorname{Dom} h_{w}$ that

$$
g\left(w_{0} u\right)=v_{0} h_{w}(u)
$$

where $w_{0}$ and $v_{0}$ are the beginnings of length $n_{w}$ of the sequences $w$ and $g(w)$, respectively.

The sets of the form $w_{0}$ Dom $h_{w}$ are open and cover the set Dom $g$. Thus there exists a finite subcover $\left\{w_{1} \operatorname{Dom} h_{1}, w_{2} \operatorname{Dom} h_{2}, \ldots, w_{m} \operatorname{Dom} h_{m}\right\}$, where $w_{i}$ are some finite sequences and $h_{i}$ are elements of the nucleus. Then the maximal length $n_{0}$ of the sequences $w_{i}$ satisfies the conditions of the lemma.

Corollary 3.3. Suppose that a recurrent self-similar semigroup $H$ acting on $\mathcal{F}$ is contracting and let $\mathcal{N}$ be its nucleus. Then for every $w \in \mathcal{F}$ there exists an idempotent $1_{U} \in H$ defined by an element of $\mathcal{N}$ such that $w$ belongs to the domain $U$ of $1_{U}$.

Proof. The space $\mathcal{F}$ is covered, by definition, by the domains of the idempotents from $H$. The domains are open, thus the space $\mathcal{F}$ is covered by domains $U_{i}$ of a finite number of idempotents $\left\{1_{U_{1}}, 1_{U_{2}}, \ldots, 1_{U_{m}}\right\} \subset H$.

There exists, by Lemma 3.2, for every $1_{U_{i}} \in H$ a number $n_{i}$ such that every path of length $n_{i}$ starting in $1_{U_{i}} \in \Gamma(H)$ ends in an element of the nucleus. Every such an end is obviously an idempotent. Let $n=\max n_{i}$. Then the sets $\sigma^{n}\left(U_{i}\right)$ and, consequently, the space $\mathcal{F}=\sigma^{n}(\mathcal{F})$ are covered by the domains of idempotents belonging to $\mathcal{N}$.

Denote by $\mathcal{G}(\mathcal{N})$ the set of germs $(h, w) \in \mathcal{G}(H)$ such that $h \in \mathcal{N}$. Then the condition in Definition 3.2 can be formulated as the requirement that $\sigma_{*}^{n}((h, w)) \in \mathcal{G}(\mathcal{N})$ for all $(h, w)$ and for all $n=n(h, w)$ big enough. Note also that the set $\mathcal{G}(\mathcal{N})$ is a compact $\sigma_{*}$-invariant subset of $\mathcal{G}(H)$. 


\subsection{Limit solenoid}

Definition 3.3. Let $H$ be a recurrent contracting self-similar semigroup, acting on a shift space $\mathcal{F}$. Let $\mathcal{N}$ be its nucleus and let $\mathcal{F}_{\mathbb{Z}}$ be the natural extension of $\mathcal{F}$. We say that two bi-infinite sequences $\left(\ldots x_{-2} x_{-1} \cdot x_{0} x_{1} \ldots\right),\left(\ldots y_{-2} y_{-1} \cdot y_{0} y_{1} \ldots\right) \in \mathcal{F}_{\mathbb{Z}}$ are asymptotically equivalent if there exists a bi-infinite path in the full automaton $\Gamma(H)$ labeled by the consecutive labels

$$
\ldots\left(x_{-2} ; y_{-2}\right),\left(x_{-1} ; y_{-1}\right),\left(x_{0} ; y_{0}\right),\left(x_{1} ; y_{1}\right) \ldots
$$

with all its vertices belonging to the nucleus $\mathcal{N}$ of the semigroup.

Lemma 3.4. Two sequences $\left(\ldots x_{-2} x_{-1} \cdot x_{0} x_{1} \ldots\right)$ and $\left(\ldots y_{-2} y_{-1} \cdot y_{0} y_{1} \ldots\right) \in \mathcal{F}_{\mathbb{Z}}$ are asymptotically equivalent if and only if for every $n \in \mathbb{Z}$ there exists $g_{n} \in \mathcal{N}$ such that

$$
g_{n}\left(x_{n} x_{n+1} \ldots\right)=y_{n} y_{n+1} \ldots
$$

Proof. The "only if" part of the lemma is trivial.

Let $A_{n} \subset \mathcal{N}$ be the set of all elements $g_{n}$ such that $g_{n}\left(x_{n} x_{n+1} \ldots\right)=y_{n} y_{n+1} \ldots$ It is a non-empty finite set, and we have a natural map $A_{n-1} \longrightarrow A_{n}$, mapping a state $g_{n}$ to the next state $g_{n-1}$ in the path defining the respective transition. In this way we get an inverse sequence of finite sets. It is well known that the inverse limit of this sequence is not empty. Any point of the inverse limit defines an infinite path labeled by the labels $\ldots\left(x_{-1} ; y_{-1}\right),\left(x_{0} ; y_{0}\right),\left(x_{1} ; y_{1}\right), \ldots$

Proposition 3.5. The asymptotic equivalence is an equivalence relation on $\mathcal{F}_{\mathbb{Z}}$ and the quotient of the space $\mathcal{F}_{\mathbb{Z}}$ by this equivalence relation is a metrizable topological space of topological dimension less than $|\mathcal{N}|$.

Proof. It is easy to prove that if $g \in \mathcal{N}$, then $g^{-1} \in \mathcal{N}$ and thus the asymptotic equivalence is symmetric. The fact that it is reflexive follows directly from Corollary 3.3 and Lemma 3.4. Let us prove that it is transitive.

Suppose that a sequence $\xi=\ldots x_{-2} x_{-1} . x_{0} x_{1} \ldots \in \mathcal{F}_{\mathbb{Z}}$ is equivalent to a sequence $\eta=$ $\ldots y_{-2} y_{-1} \cdot y_{0} y_{1} \ldots \in \mathcal{F}_{\mathbb{Z}}$ and $\eta$ is equivalent to $\zeta=\ldots z_{-2} z_{-1} \cdot z_{0} z_{1} \ldots \in \mathcal{F}_{\mathbb{Z}}$. Let $\left(\ldots g_{-1}, g_{0}, g_{1} \ldots\right)$ be such a sequence of elements of $\mathcal{N}$ that $g_{n}\left(x_{n} x_{n+1} \ldots\right)=y_{n} y_{n+1} \ldots$ and let $\left(\ldots h_{-1}, h_{0}, h_{1} \ldots\right)$ be such a sequence of elements of $\mathcal{N}$ that $h_{n}\left(y_{n} y_{n+1} \ldots\right)=z_{n} z_{n+1} \ldots$ Then

$$
h_{n} g_{n}\left(x_{n} x_{n+1} \ldots\right)=z_{n} z_{n+1} \ldots
$$

There exists, by Lemma 3.2, a number $n_{0} \in \mathbb{N}$ such that every path of the length $\geq n_{0}$ in $\Gamma(H)$, starting in a product of two elements of $\mathcal{N}$, ends in $\mathcal{N}$. This implies that the transition $\left(x_{n} x_{n+1} \ldots ; z_{n} z_{n+1} \ldots\right)$ is defined by an element of the nucleus for every $n \in \mathbb{Z}$ and the sequences $\xi$ and $\zeta$ are equivalent by Lemma 3.4.

It follows directly from the definitions that the asymptotic equivalence relation is closed (since even its graph is closed). It follows also that for every sequence $\xi=\ldots x_{-2} x_{-1} \cdot x_{0} x_{1} \ldots \in \mathcal{F}_{\mathbb{Z}}$ and for every $n \in \mathbb{Z}$ the number of possible tails $y_{n} y_{n-1} \ldots$ of a sequence $\ldots y_{-2} y_{-1} \cdot y_{0} y_{1} \ldots$ asymptotically equivalent to $\xi$ is not greater than $|\mathcal{N}|$. This implies that every asymptotic equivalence class on $\mathcal{F}_{\mathbb{Z}}$ has not more than $|\mathcal{N}|$ elements.

Now by Theorem 4.2.13 from [Eng77], the quotient space is metrizable, since it is a quotient of a compact separable metrizable space $\mathcal{F}_{\mathbb{Z}}$ by a closed equivalence relation with compact equivalence classes. The assertion about the dimension follows from Hurewicz formula (see [Kur61] page $52)$.

Definition 3.4. The quotient of the space $\mathcal{F}_{\mathbb{Z}}$ by the asymptotic equivalence relation is called the limit solenoid of the self-similar semigroup and is denoted $\mathcal{S}_{H}$.

The shift $\sigma_{\mathbb{Z}}: \mathcal{F}_{\mathbb{Z}} \longrightarrow \mathcal{F}_{\mathbb{Z}}$ induces a homeomorphism of the limit solenoid $\mathcal{S}_{H}$, since the asymptotic equivalence relation agrees with the shift. Let us denote this homeomorphism by $\mathrm{s}$. We have associated in this way a dynamical system $\left(\mathcal{S}_{H}, \mathrm{~s}\right)$ with every contracting recurrent action of an inverse semigroup. 
Definition 3.5. A tile $\mathcal{T}_{w}$ corresponding to a sequence $w \in \mathcal{F}$ is the image of the set of the sequences of the form $\ldots x_{-2} x_{-1} \cdot w \in \mathcal{F}_{\mathbb{Z}}$ in the solenoid $\mathcal{S}_{H}$. A leaf of the solenoid is the union of all the tiles $\mathcal{T}_{w}$, for which $w$ belongs to a given $H$-orbit.

Every tile $\mathcal{T}_{w}$ is a closed subset of $\mathcal{S}_{H}$, and the solenoid $\mathcal{S}_{H}$ is a disjoint union of the leaves.

It follows directly from the definition that

$$
\mathbf{s}\left(\mathcal{T}_{w}\right)=\bigcup_{x \in X} \mathcal{T}_{x w}
$$

where $\mathcal{T}_{x w}$ is considered to be empty if $x w \notin \mathcal{F}$. This relation is similar to the inflation of substitution tilings, see [AP98].

\subsection{Examples}

Adding machine. The nucleus of the adding machine action consists of the states (vertices) $a, 1, a^{-1}$ (see [BGN03]). It follows directly from the diagram of the nucleus that two sequences $\left(\ldots x_{-2} x_{-1} \cdot x_{0} x_{1} \ldots\right),\left(\ldots y_{-2} y_{-1} \cdot y_{0} y_{1} \ldots\right) \in\{0,1\}^{\mathbb{Z}}$ are asymptotically equivalent if and only if they are either equal or are of the form $\left(\ldots 0001 a_{n} a_{n+1} \ldots\right),\left(\ldots 1110 a_{n} a_{n+1}\right)$. But this is the usual identification of the reals written in the binary numeration system. More precisely, the sequences are asymptotically equivalent if and only if

$$
\sum_{n=0}^{+\infty} x_{n} \cdot 2^{n}-\sum_{n=0}^{+\infty} y_{n} \cdot 2^{n}=\sum_{n=-\infty}^{-1} y_{n} \cdot 2^{n}-\sum_{n=-\infty}^{-1} y_{n} \cdot 2^{n},
$$

where the left-hand side of the equality is computed in the ring $\mathbb{Z}_{2}$ of dyadics, the right-hand side in the field $\mathbb{R}$ of real numbers, but both sides must belong to $\mathbb{Z}$ and be equal.

The limit solenoid of the adding machine action is then the well known Smale solenoid, homeomorphic to the inverse limit of the circle $\mathbb{R} / \mathbb{Z}$ with respect to the iterations of its double selfcovering $f: t \mapsto 2 t$ (see, for example [Put96] and [BGN03]). The respective shift is induced by $f$ on the inverse limit.

This solenoid $\mathcal{S}_{\mathbb{Z}}$ can be also constructed in the following way. Take the direct product $[0,1] \times X^{\mathbb{N}}$ of the real segment by the Cantor space $X^{\mathbb{N}}=\{0,1\}^{\mathbb{N}}$ and identify every point $(1, w)$ with the point $(0, a(w))$, where $a$ is the adding machine. The obtained space is homeomorphic to the limit solenoid $\mathcal{S}_{\mathbb{Z}}$ of the adding machine action. This follows easily from (1).

Penrose tilings. The self-similar semigroup associated with the Penrose tiling is contracting. The tiled leaves of its limit solenoid are exactly the Penrose tilings. (Except for the tilings having a non-trivial symmetry group; then the respective leaf of the solenoid is the quotient of the tiling by its symmetry group.)

The limit solenoid is homeomorphic to the space of Penrose tilings with marked points. Two marked Penrose tilings are close in this space if the tilings can be superimposed by an affine transformation in such a way that the marked points will be close to each other and the tilings agree on a large ball around one of the marked point. For an exact definition of this space, see the paper [AP98]. Note, however, that we do not care about the orientation of the tiles. Respectively, our space is a finite-to-one quotient of the space defined in [AP98].

The fact that the semigroup is contracting and the above description of the limit space follow easily from Theorem 4.6 below.

\section{Smale spaces}

\subsection{Definitions}

Let us define the notion of a Smale space, following [Rue78] and [Put96]. Let $(\Omega, d)$ be a compact metric space together with a homeomorphism $f: \Omega \longrightarrow \Omega$. We require that there exists a number 
$\epsilon_{0}>0$ and a continuous function (canonical coordinates):

$$
[\cdot, \cdot]:\left\{(\xi, \eta) \in \Omega \times \Omega: d(\xi, \eta)<\epsilon_{0}\right\} \longrightarrow \Omega
$$

such that

$$
[\xi, \xi]=\xi, \quad[[\xi, \eta], \zeta]=[\xi, \zeta], \quad[\xi,[\eta, \zeta]]=[\xi, \zeta]
$$

for all $\xi, \eta, \zeta \in \Omega$ for which the respective expressions are defined.

Define for every $0<\epsilon \leq \epsilon_{0}$

$$
\begin{aligned}
& W_{\epsilon}^{s}(\xi)=\{\eta \in \Omega:[\xi, \eta]=\eta, \quad d(\xi, \eta)<\epsilon\} \\
& W_{\epsilon}^{u}(\xi)=\{\eta \in \Omega:[\eta, \xi]=\eta, \quad d(\xi, \eta)<\epsilon\} .
\end{aligned}
$$

We require that

$$
f([\xi, \eta])=[f(\xi), f(\eta)],
$$

whenever both sides of the equality are defined and that

$$
\begin{aligned}
d(f(\eta), f(\zeta)) & \leq \lambda_{0} d(\eta, \zeta), & & \eta, \zeta \in W_{\epsilon}^{s}(\xi) \\
d\left(f^{-1}(\eta), f^{-1}(\zeta)\right) & \leq \lambda_{0} d(\eta, \zeta), & & \eta, \zeta \in W_{\epsilon}^{u}(\xi)
\end{aligned}
$$

for some fixed $0<\lambda_{0}<1$.

Then we have for every $\xi \in \Omega$ a homeomorphism

$$
[\cdot, \cdot]: W_{\epsilon_{0} / 2}^{u}(\xi) \times W_{\epsilon_{0} / 2}^{s}(\xi) \longrightarrow \Omega
$$

onto a neighborhood of $\xi$, and

$$
\{[\xi, \eta]\}=W_{\epsilon_{0}}^{s}(\xi) \cap W_{\epsilon_{0}}^{u}(\eta) .
$$

In general, a subset $R \subset \Omega$ is called rectangle, if its diameter is less than $\epsilon_{0}$ and for every point $\xi \in R$ the map

$$
[\cdot, \cdot]: W_{\epsilon_{0}}^{u}(\xi, R) \times W_{\epsilon_{0}}^{s}(\xi, R) \longrightarrow R
$$

is a homeomorphism, where $W_{\epsilon_{0}}^{u}(\xi, R)=W_{\epsilon_{0}}^{u}(\xi) \cap R$ and $W_{\epsilon_{0}}^{s}(\xi, R)=W_{\epsilon_{0}}^{s}(\xi) \cap R$.

The next lemma follows directly from the definition.

Lemma 4.1. If $R$ is a rectangle and $\xi_{1}, \xi_{2} \in R$, then the map

$$
W_{\epsilon_{0}}^{s}\left(\xi_{1}, R\right) \longrightarrow W_{\epsilon_{0}}^{s}\left(\xi_{2}, R\right): \eta \mapsto\left[\xi_{2}, \eta\right] .
$$

is a homeomorphism. Similarly, the map $\eta \mapsto\left[\eta, \xi_{2}\right]$ is a homeomorphism from $W_{\epsilon_{0}}^{u}\left(\xi_{1}, R\right)$ to $W_{\epsilon_{0}}^{u}\left(\xi_{2}, R\right)$.

A Markov partition of a Smale space (see [Bow75]) is a finite cover $\mathcal{M}$ of the space $\Omega$ by closed proper rectangles with disjoint interiors (a rectangle $R$ is said to be proper if $R=\overline{\operatorname{int} R}$ ), such that if $\xi \in \operatorname{int} R$ and $f(\xi) \in \operatorname{int} R^{\prime}$ for $R, R^{\prime} \in \mathcal{M}$, then

$$
f\left(W_{\epsilon_{0}}^{s}(\xi, R)\right) \subseteq R^{\prime} \quad \text { and } \quad f^{-1}\left(W_{\epsilon_{0}}^{u}\left(f(\xi), R^{\prime}\right)\right) \subseteq R
$$

If $R$ is a closed rectangle, then (see [Bow75]) its boundary $\partial R$ is a union $\partial^{s} R \cup \partial^{u} R$, where

$$
\begin{aligned}
& \partial^{s} R=\left\{\xi \in R: \xi \notin \operatorname{int} W_{\epsilon_{0}}^{u}(\xi, R)\right\}, \\
& \partial^{u} R=\left\{\xi \in R: \xi \notin \operatorname{int} W_{\epsilon_{0}}^{s}(\xi, R)\right\},
\end{aligned}
$$

where the interiors of $W_{\epsilon_{0}}^{u}(\xi, R)$ and $W_{\epsilon_{0}}^{s}(\xi, R)$ are taken as in subsets of the topological spaces $W_{\epsilon_{0}}^{u}(\xi)$ and $W_{\epsilon_{0}}^{s}(\xi)$, respectively.

Definition 4.1. We say that a Markov partition $\mathcal{M}$ is s-resolved if for every rectangle $R \in \mathcal{M}$ and $\xi \in R$ the set $W_{\epsilon_{0}}^{s}(\xi, R)$ is open in $W_{\epsilon_{0}}^{s}(\xi)$, i.e., if we have

$$
\partial R=\partial^{s} R \text {. }
$$




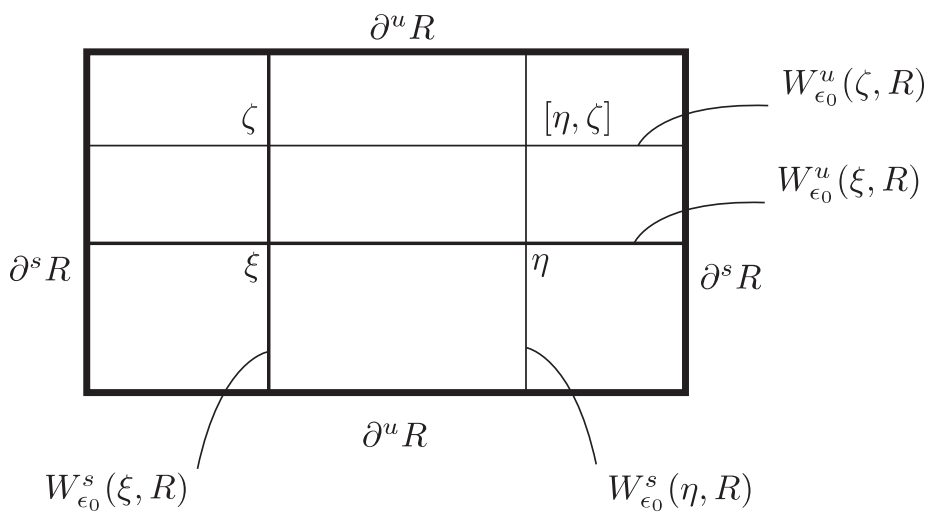

Figure 6: A rectangle.

The set $W_{\epsilon_{0}}^{s}(\xi, R)$ is always closed ,since $R$ is closed, $W_{\epsilon_{0}}^{s}(\xi, R)=\{\eta \in R:[\xi, \eta]=\eta\}$ and the map $[\cdot, \cdot]$ is continuous. Hence, if the Markov partition is $s$-resolved, then the sets $W_{\epsilon_{0}}^{s}(\xi, R)$ are clopen in $W_{\epsilon_{0}}^{s}(\xi)$ (and totally disconnected, as we will see later).

Fix an alphabet $X$ together with a one-to-one correspondence $a \mapsto R_{a}$ with the elements of a Markov partition $\mathcal{M}$ of a Smale space $(\Omega, d, f)$. Then for every point $\xi \in \Omega$ its code is a sequence $\left(\ldots a_{-2} a_{-1} \cdot a_{0} a_{1} \ldots\right) \in X^{\mathbb{Z}}$ such that for every $n \in \mathbb{N}$ we have

$$
\xi \in \bigcap_{-n \leq k \leq n} f^{k}\left(R_{a_{k}}\right)=C_{n}
$$

and the set $C_{n}$ has a non-empty interior.

Note that the diameter of the set $C_{n}$ is less than $\lambda_{0}^{n} \epsilon_{0}$. Consequently, there is not more than one point having a given code. On the other hand, one point can have different codes, since the elements of the Markov partition can have non-empty intersections.

The following result is classical.

Lemma 4.2. The set $\mathcal{F}_{\mathbb{Z}}$ of all codes of points $\xi \in \Omega$ is equal to the set of all sequences $\left(\ldots a_{-2} a_{-1} . a_{0} a_{1} \ldots\right) \in X^{\mathbb{Z}}$ such that the set int $R_{a_{k}} \cap \operatorname{int} f\left(R_{a_{k+1}}\right)$ is not empty for all $k \in \mathbb{Z}$.

The map $\pi: \mathcal{F}_{\mathbb{Z}} \longrightarrow \Omega$, putting into correspondence to a code the respective point of $\Omega$ is a continuous surjection such that

$$
f(\pi(w))=\pi\left(\sigma_{\mathbb{Z}}(w)\right) .
$$

Consequently, the set $\mathcal{F}_{\mathbb{Z}}$ is a Markov shift space. We denote by $\mathcal{F}$ the respective one-sided shift.

The following lemma follows directly from the definitions.

Lemma 4.3. If $\pi\left(\ldots a_{-2} a_{-1} \cdot a_{0} a_{1} \ldots\right)=\xi$ and $\pi\left(\ldots b_{-2} b_{-1} \cdot b_{0} b_{1} \ldots\right)=\eta$, where $a_{0}=b_{0}$ and $\xi, \eta \in R_{a_{0}}$, then $\pi\left(\ldots a_{-2} a_{-1} . b_{0} b_{1} \ldots\right)=[\xi, \eta]$. In particular, the set $W_{\epsilon_{0}}^{s}\left(R_{a_{0}}, \xi\right)$ coincides with the set of points having codes of the form $\left(\ldots a_{-2} a_{-1} \cdot a_{0} x_{1} x_{2} \ldots\right)$.

Let us prove the following fact about $s$-resolved Markov partitions.

Lemma 4.4. Let $\mathcal{M}$ be an s-resolved Markov partition of a Smale space $(\Omega, d, f)$. For every $R_{a_{0}} \in \mathcal{M}$ and every point $\xi \in R_{a_{0}}$ having a code $\left(\ldots a_{-2} a_{-1} . a_{0} a_{1} \ldots\right)$, the restriction of $\pi$ onto the set $\mathcal{F}^{s}(\xi)$ of sequences of the form $\ldots a_{-2} a_{-1} . a_{0} x_{1} x_{2} \ldots \in \mathcal{F}_{\mathbb{Z}}$ is a homeomorphism onto the space $W_{\epsilon_{0}}^{s}\left(\xi, R_{a_{0}}\right)$.

Proof. It is sufficient to prove that the restriction of the map $\pi$ onto the set $\mathcal{F}^{s}(\xi)$ is injective, since it is surjective by Lemma 4.3 and the spaces $\mathcal{F}^{s}(\xi)$ and $W_{\epsilon_{0}}^{s}\left(\xi_{0}, R_{a_{0}}\right)$ are compact. 
Suppose at first that $\xi \in \operatorname{int} R_{a_{0}}$. Then $W_{\epsilon_{0}}^{s}\left(\xi, R_{a_{0}}\right) \subset \operatorname{int} R_{a_{0}}$, by definition of an $s$-resolved Markov partition. Let $\eta \in W_{\epsilon_{0}}^{s}\left(\xi, R_{a_{0}}\right)$ be an arbitrary point and let $\left(\ldots a_{-2} a_{-1} . a_{0} b_{0} b_{1} \ldots\right)$ be its code. The point $\eta$ is an internal point of $R_{a_{0}}$ and thus it is an internal point of $W_{\epsilon_{0}}^{u}\left(\eta, R_{a_{0}}\right)$. Then for every $n \in \mathbb{N}$ the point $f^{-n}(\eta)$ is an internal point of the set $f^{-n}\left(W_{\epsilon_{0}}^{u}\left(\eta, R_{a_{0}}\right)\right) \subset R_{b_{n}}$, and thus is an internal point of $R_{b_{n}}$, by definition of an $s$-resolved Markov partition. Consequently, the point $\eta$ belongs only to the rectangle $R_{b_{n}}$ and the sequence $b_{1} b_{2} \ldots$ is uniquely determined by $\left(\ldots a_{-2} a_{-1} a_{0}\right)$ and $\eta$, i.e., the restriction of the map $\pi$ onto $\mathcal{F}^{s}(\xi)$ is injective.

For other points of $R_{a_{0}}$ one needs just to apply Lemma 4.1 and Lemma 4.3.

\subsection{Adjacency semigroup}

Let us fix now some $s$-resolved Markov partition $\mathcal{M}=\left\{R_{a}\right\}_{a \in X}$ of a Smale space $(\Omega, d, f)$.

Let $a_{0}, b_{0} \in X$ be arbitrary letters such that the intersection $R_{a_{0}} \cap R_{b_{0}}$ is not empty, and let $\xi \in$ $R_{a_{0}} \cap R_{b_{0}}$. Then the point $\xi$ has codes of the form $\left(\ldots a_{-2} a_{-1} . a_{0} a_{1} \ldots\right)$ and $\left(\ldots b_{-2} b_{-1} . b_{0} b_{1} \ldots\right)$.

Define now a partial transformation $T_{\left(a_{0} ; b_{0}\right)}$ of the space $\mathcal{F}$, which maps a sequence $a_{0} x_{1} x_{2} \ldots \in$ $\mathcal{F}$ to a sequence $b_{0} y_{1} y_{2} \ldots \in \mathcal{F}$ if and only if

$$
\pi\left(\ldots a_{-2} a_{-1} \cdot a_{0} x_{1} x_{2} \ldots\right)=\pi\left(\ldots b_{-2} b_{-1} \cdot b_{0} y_{1} y_{2} \ldots\right) .
$$

Proposition 4.5. The transformation $T_{\left(a_{0} ; b_{0}\right)}$ is a well defined local homeomorphism of the space $\mathcal{F}$ depending only on the letters $a_{0}$ and $b_{0}$.

Proof. Let us prove that the transformation $T_{\left(a_{0} ; b_{0}\right)}$ does not depend on the choice of the point $\xi$ and its codes $w_{1}=\left(\ldots a_{-2} a_{-1} \cdot a_{0} a_{1} \ldots\right), w_{2}=\left(\ldots b_{-2} b_{-1} \cdot b_{0} b_{1} \ldots\right)$. Let

$$
w_{1}^{\prime}=\left(\ldots a_{-2}^{\prime} a_{-1}^{\prime} \cdot a_{0}^{\prime} a_{1}^{\prime} \ldots\right), \quad w_{2}^{\prime}=\left(\ldots b_{-2}^{\prime} b_{-1}^{\prime} \cdot b_{0}^{\prime} b_{1}^{\prime} \ldots\right) \in \mathcal{F}
$$

be codes of a point $\xi^{\prime}$ such that $a_{0}^{\prime}=a_{0}$ and $b_{0}^{\prime}=b_{0}$.

Let $\left(\ldots a_{-2} a_{-1} . a_{0} x_{1} x_{2} \ldots\right)$ and $\left(\ldots b_{-2} b_{-1} . b_{0} y_{1} y_{2} \ldots\right)$ be codes of a point $\zeta$. Then, by Lemma 4.3 , the sequences $\left(\ldots a_{-2}^{\prime} a_{-1}^{\prime} . a_{0}^{\prime} x_{1} x_{2} \ldots\right)$ and $\left(\ldots b_{-2}^{\prime} b_{-1}^{\prime} . b_{0}^{\prime} y_{1} y_{2} \ldots\right)$ are codes of the point $\left[\xi^{\prime}, \zeta\right]$. Hence, the transformation $T_{\left(a_{0} ; b_{0}\right)}$ defined by $\xi, w_{1}$ and $w_{2}$ is the same as the transformation defined by $\xi^{\prime}, w_{1}^{\prime}$ and $w_{2}^{\prime}$.

Let us prove that the transformation $T_{\left(a_{0} ; b_{0}\right)}$ is a local homeomorphism (in particular, that it is a well defined map). Let $\xi \in R_{a_{0}} \cap R_{b_{0}}$ be a point, and let $\left(\ldots a_{-2} a_{-1} \cdot a_{0} a_{1} \ldots\right)$ and $\left(\ldots b_{-2} b_{-1} . b_{0} b_{1} \ldots\right)$ be codes of the points $\xi$. Recall that $\mathcal{F}^{s}(\xi)$ denotes the set of sequences of the form $\left(\ldots a_{-2} a_{-1} \cdot a_{0} x_{1} x_{2} \ldots\right) \in \mathcal{F}$. The map

$$
\rho_{\xi}: a_{0} x_{1} x_{2} \ldots \mapsto \ldots a_{-2} a_{-1} \cdot a_{0} x_{1} x_{2} \ldots
$$

is a homeomorphism from the space $\mathcal{F}_{a_{0}}$ of all the sequences belonging to $\mathcal{F}$ and starting with $a_{0}$ to the set $\mathcal{F}^{s}(\xi)$. Let us denote by $\pi_{a_{0}, \xi}$ the homeomorphism $\left.\pi\right|_{\mathcal{F}^{s}(\xi)} \circ \rho_{\xi}: \mathcal{F}_{a_{0}} \longrightarrow W_{\epsilon_{0}}^{s}\left(\xi, R_{a_{0}}\right)$. It is a homeomorphism by Lemma 4.4 .

Denote

$$
\begin{aligned}
& D_{a_{0}}=\pi_{a_{0}, \xi}^{-1}\left(W_{\epsilon_{0}}^{s}\left(\xi, R_{a_{0}}\right) \cap W_{\epsilon_{0}}^{s}\left(\xi, R_{b_{0}}\right)\right) \\
& D_{b_{0}}=\pi_{b_{0}, \xi}^{-1}\left(W_{\epsilon_{0}}^{s}\left(\xi, R_{a_{0}}\right) \cap W_{\epsilon_{0}}^{s}\left(\xi, R_{b_{0}}\right)\right) .
\end{aligned}
$$

Then, by Lemma 4.4 the set $D_{a_{0}}$ is the domain of the map $T_{\left(a_{0} ; b_{0}\right)}$, and

$$
T_{\left(a_{0} ; b_{0}\right)}=\left.\left(\left.\pi\right|_{D_{a_{0}}}\right)^{-1} \circ \pi\right|_{D_{b_{0}}},
$$

so that $T_{\left(a_{0} ; b_{0}\right)}$ is a local homeomorphism.

Note that the transformation $T_{\left(a_{0} ; a_{0}\right)}$ is the idempotent with domain $\mathcal{F}_{a_{0}}$.

Definition 4.2. The adjacency semigroup of an s-resolved Markov partition is the inverse semigroup generated by the set of partial homeomorphisms $\mathcal{T}=\left\{T_{\left(a_{0} ; b_{0}\right)}: R_{a_{0}} \cap R_{b_{0}} \neq \varnothing\right\}$ of the space $\mathcal{F}$. 
The groupoid of germs of the adjacency semigroup can be interpreted as the holonomy groupoid of the unstable foliation coming from the local structure of a direct product on the Smale space. See [Hae01] for the definition of the holonomy groupoid of a foliation.

Theorem 4.6. The adjacency semigroup $H$ of a Smale space $(\Omega, f)$ is self-similar and contracting. Its limit dynamical system $\left(\mathcal{S}_{H}, \mathrm{~s}\right)$ is topologically conjugate to the system $(\Omega, f)$. In particular, the limit solenoid $\mathcal{S}_{H}$ is homeomorphic to $\Omega$.

Proof. Let us prove that the semigroup $H$ is self-similar. We shall construct the automaton generating the semigroup $H$ and use then Proposition 2.2.

Let us construct a directed graph $\Gamma$ with the vertices $\mathcal{T}$ and connect the vertex $T_{\left(a_{0} ; b_{0}\right)}$ to the vertex to $T_{\left(a_{1} ; b_{1}\right)}$ by an arrow labeled by $\left(a_{0} ; b_{0}\right)$ if and only if there exists a pair of sequences

$$
\left(\ldots a_{-2} a_{-1} \cdot a_{0} a_{1} a_{2} \ldots\right), \quad\left(\ldots b_{-2} b_{-1} \cdot b_{0} b_{1} b_{2} \ldots\right) \in \mathcal{F}_{\mathbb{Z}}
$$

representing the same point of the Smale space $\Omega$.

Let us prove that the constructed automaton defines the transformations $T_{(a ; b)}$ and thus generates the semigroup $H$.

Let $T_{\left(a_{0} ; b_{0}\right)}$ be an element of $\mathcal{T}$ and let $T_{\left(a_{1}^{(k)} ; b_{1}^{(k)}\right)}, k=1, \ldots, m$, be all the ends of the arrows starting in $T_{\left(a_{0} ; b_{0}\right)}$. Then for every $k$ there exists a pair of codes

$$
\left(\ldots a_{-2}^{(k)} a_{-1}^{(k)} \cdot a_{0}^{(k)} a_{1}^{(k)} a_{2}^{(k)} \ldots\right), \quad\left(\ldots b_{-2}^{(k)} b_{-1}^{(k)} \cdot b_{0}^{(k)} b_{1}^{(k)} b_{2}^{(k)} \ldots\right) \in \mathcal{F}_{\mathbb{Z}},
$$

of a point $\xi_{k} \in \Omega$ such that $a_{0}^{(k)}=a_{0}$ and $b_{0}^{(k)}=b_{0}$. It follows directly from the definition of the transformation $T_{\left(a_{1}^{(k)} ; b_{1}^{(k)}\right)}$ (and the fact that it is well defined) that the condition $a_{1}^{(k)} x_{2} x_{3} \ldots \in$ $\operatorname{Dom} T_{\left(a_{1}^{(k)} ; b_{1}^{(k)}\right)}$ is equivalent to the condition $a_{0} a_{1}^{(k)} x_{2} x_{3} \ldots \in \operatorname{Dom} T_{\left(a_{0} ; b_{0}\right)}$ and that the equality

$$
T_{\left(a_{0} ; b_{0}\right)}\left(a_{0} a_{1}^{(k)} x_{2} x_{3} \ldots\right)=b_{0} T_{\left(a_{1}^{(k)} ; b_{1}^{(k)}\right)}\left(a_{1}^{(k)} x_{2} x_{3} \ldots\right)
$$

holds. In particular, we have

$$
\operatorname{Dom} T_{\left(a_{0} ; b_{0}\right)}=\bigsqcup_{k=1, \ldots, m} a_{0} \operatorname{Dom} T_{\left(a_{1}^{(k)} ; b_{1}^{(k)}\right)} .
$$

The union is disjoint, since every sequence $a_{0} a_{1} \ldots \in \operatorname{Dom} T_{\left(a_{0} ; b_{0}\right)}$ belongs only to $a_{0} \operatorname{Dom} T_{\left(a_{1} ; b_{1}\right)}$, where $b_{1}$ is the second letter of the sequence $T_{\left(a_{0} ; b_{0}\right)}\left(a_{0} a_{1} \ldots\right)$.

Repeating now the arguments from the proof of Proposition 2.1, we deduce from (4) and (5) that the state $T_{\left(a_{0} ; b_{0}\right)}$ of the automaton $\Gamma$ defines the transformation $T_{\left(a_{0} ; b_{0}\right)}$.

Let us prove that the semigroup $H$ is contracting. We need at first some auxiliary definitions and facts.

Let $R_{1}, R_{2}, R_{3}$ be three rectangles from the Markov partition, and denote by $\Xi_{2}$ the set all $\xi_{2} \in R_{2}$ such that there exist $\xi_{1} \in R_{1}$ and $\xi_{3} \in R_{3}$ such that

$$
W_{\epsilon_{0}}^{u}\left(\xi_{1}, R_{1}\right) \cap W_{\epsilon_{0}}^{u}\left(\xi_{2}, R_{2}\right) \neq \varnothing, \quad W_{\epsilon_{0}}^{u}\left(\xi_{2}, R_{2}\right) \cap W_{\epsilon_{0}}^{u}\left(\xi_{3}, R_{3}\right) \neq \varnothing .
$$

Note that the sets $W_{\epsilon_{0}}^{u}\left(\xi_{1}, R_{1}\right)$ and $W_{\epsilon_{0}}^{u}\left(\xi_{3}, R_{3}\right)$ depend only on $W_{\epsilon_{0}}^{u}\left(\xi_{2}, R_{3}\right), R_{1}, R_{2}, R_{3}$.

It is easy to see that the set $\Xi_{2}$ is a closed rectangle and is the image of the set $W_{\epsilon_{0}}^{u}\left(\xi_{2}, R_{2}\right) \times S$ under the homeomorphism $[\cdot, \cdot]$, where $S$ is a closed subset of $W_{\epsilon_{0}}^{s}\left(\xi_{2}, R_{2}\right)$ (see Figure 7).

The set

$$
\left(\Xi_{2} \cap R_{1}\right) \cap\left(\Xi_{2} \cap R_{3}\right)
$$

is also a rectangle and is equal to the image of the set $U\left(\xi_{2}\right) \times S$ under the homeomorphism $[\cdot, \cdot]$, where

$$
U\left(\xi_{2}\right)=W_{\epsilon_{0}}^{u}\left(\xi_{1}, R_{1}\right) \cap W_{\epsilon_{0}}^{u}\left(\xi_{2}, R_{2}\right) \cap W_{\epsilon_{0}}^{u}\left(\xi_{3}, R_{3}\right),
$$




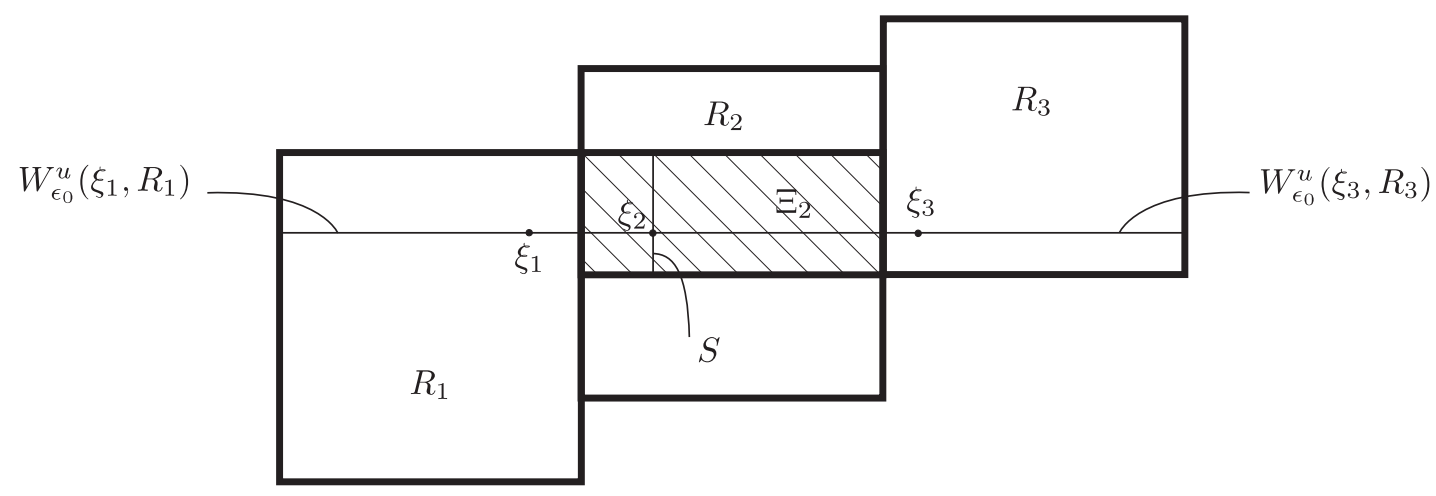

Figure 7: The set $\Xi_{2}$.

and $\xi_{1}, \xi_{3}$ are some points satisfying conditions (6). In particular, the set $U\left(\xi_{2}\right)$ does not depend, up to a homeomorphism on $\xi_{2} \in \Xi_{2}$.

Define $c\left(R_{1}, R_{2}, R_{3}\right)$ to be equal to the distance between the compact sets $\Xi_{2} \cap R_{1}$ and $\Xi_{2} \cap R_{3}$, if they are disjoint, i.e., if the set $U\left(\xi_{2}\right)$ (for some, and thus for all $\xi_{2} \in \Xi_{2}$ ) is empty. Let $c$ be the minimal among the defined numbers $c\left(R_{1}, R_{2}, R_{3}\right)$ for all possible triples of rectangles $R_{1}, R_{2}, R_{3} \in \mathcal{M}$. If the set of the defined numbers $c\left(R_{1}, R_{2}, R_{3}\right)$ is empty, then we choose $c$ to be any positive number.

The positive number $c$ is so defined that if $R_{1}, R_{2}, R_{3} \in \mathcal{M}$ and $\xi_{i} \in R_{i}$ are such that $\xi_{1}, \xi_{2} \in R_{2}$, conditions (6) hold and $d\left(\xi_{1}, \xi_{3}\right)<c$, then $W_{\epsilon_{0}}^{s}\left(\xi_{1}, R_{1}\right) \cap W_{\epsilon_{0}}^{s}\left(\xi_{2}, R_{2}\right) \cap W_{\epsilon_{0}}^{s}\left(\xi_{3}, R_{3}\right) \neq \varnothing$.

Let now $T_{\left(b_{0} ; c_{0}\right)}, T_{\left(a_{0} ; b_{0}\right)}$ be two generators of the adjacency semigroup $H$, and sequences $a_{0} a_{1} \ldots, b_{0} b_{1} \ldots, c_{0} c_{1} \ldots \in \mathcal{F}$ are such that $a_{0} a_{1} \ldots \in \operatorname{Dom}\left(T_{\left(b_{0} ; c_{0}\right)} \cdot T_{\left(a_{0} ; b_{0}\right)}\right)$ and

$$
\begin{aligned}
& b_{0} b_{1} \ldots=T_{\left(a_{0} ; b_{0}\right)}\left(a_{0} a_{1} \ldots\right), \\
& c_{0} c_{1} \ldots=T_{\left(b_{0} ; c_{0}\right)}\left(b_{0} b_{1} \ldots\right) .
\end{aligned}
$$

Then there exist sequences from $\mathcal{F}_{\mathbb{Z}}$ and points of $\Omega$ such that

$$
\begin{aligned}
& \xi=\pi\left(\ldots a_{-2} a_{-1} \cdot a_{0} a_{1} a_{2} \ldots\right)=\pi\left(\ldots b_{-2} b_{-1} \cdot b_{0} b_{1} b_{2} \ldots\right) \\
& \eta=\pi\left(\ldots b_{-2}^{\prime} b_{-1}^{\prime} \cdot b_{0} b_{1} b_{2} \ldots\right)=\pi\left(\ldots c_{-2} c_{-1} \cdot c_{0} c_{1} c_{2} \ldots\right) .
\end{aligned}
$$

The definition of a Smale space implies than for every $n>\frac{\log \left(c / \operatorname{Diam} R_{b_{0}}\right)}{-\log \lambda_{0}}$ the inequality $d\left(f^{-n}(\xi), f^{-n}(\eta)\right)<c$ holds. We have $f^{-n}(\xi) \in R_{a_{n}} \cap R_{b_{n}}$ and $f^{-n}(\eta) \in R_{b_{n}} \cap R_{c_{n}}$. We also have $W_{\epsilon_{0}}^{u}\left(\xi, R_{b_{n}}\right)=W_{\epsilon_{0}}^{u}\left(\eta, R_{b_{n}}\right)$. Then it follows from the choice of the constant $c$ that the intersection $W_{\epsilon_{0}}^{u}\left(\xi, R_{a_{n}}\right) \cap W_{\epsilon_{0}}^{u}\left(\eta, R_{c_{n}}\right)$ is not empty. This implies that $T_{\left(a_{n} ; c_{n}\right)}\left(a_{n} a_{n+1} \ldots\right)=c_{n} c_{n+1} \ldots$ and that the beginning of length $n$ of the path starting in $T_{\left(b_{0} ; c_{0}\right)} \cdot T_{\left(a_{0} ; b_{0}\right)}$ and defining the transition $\left(a_{0} a_{1} \ldots ; c_{0} c_{1} \ldots\right)$ ends in $T_{\left(a_{n} ; c_{n}\right)}$.

We have proved that a path in the full automaton of the adjacency semigroup which starts in a product of two generators is eventually trapped in the set $\mathcal{T}=\left\{T_{(a ; b)}: a, b \in X\right\}$. But it follows then by induction that the path starting in a product of any number of generators is also eventually trapped in the set $\mathcal{T}$. This shows that the semigroup $H$ is contracting with the nucleus a subset of $\mathcal{T}$.

Let us prove that the dynamical systems $(\Omega, f)$ and $\left(\mathcal{S}_{H}, \mathrm{~s}\right)$ are topologically conjugate. Both $\Omega$ and $\mathcal{S}_{H}$ are quotients of the topological space $\mathcal{F}_{\mathbb{Z}}$. It is sufficient to prove that the respective equivalence relations are equal. Then the conjugacy of the dynamical systems will follow automatically, due to the definition of the map s and Lemma 4.2.

Let $w_{1}=\left(\ldots a_{-2} a_{-1} . a_{0} a_{1} \ldots\right)$ and $w_{2}=\left(\ldots b_{-2} b_{-1} . b_{0} b_{1} \ldots\right)$ be two points, which are asymptotically equivalent in $\mathcal{F}_{\mathbb{Z}}$ with respect to the action of the semigroup $H$. Then for every 
$n \in \mathbb{Z}$ there exists a pair of sequences

$$
w_{1}^{(n)}=\left(\ldots a_{-2}^{(n)} a_{-1}^{(n)} \cdot a_{0}^{(n)} a_{1}^{(n)} \ldots\right), \quad w_{2}^{(n)}=\left(\ldots b_{-2}^{(n)} b_{-1}^{(n)} \cdot b_{0}^{(n)} b_{1}^{(n)} \ldots\right) \in \mathcal{F}_{\mathbb{Z}}
$$

such that $\pi\left(w_{1}^{(n)}\right)=\pi\left(w_{2}^{(n)}\right)$ and $a_{k}=a_{k}^{(n)}, b_{k}=b_{k}^{(n)}$ for all $k \geq n$ (we used here the fact that the nucleus of $H$ is a subset of $\mathcal{T}$ ). Then

$$
\pi\left(w_{1}\right)=\pi\left(\lim _{n \rightarrow-\infty} w_{1}^{(n)}\right)=\lim _{n \rightarrow-\infty} \pi\left(w_{1}^{(n)}\right)=\lim _{n \rightarrow-\infty} \pi\left(w_{2}^{(n)}\right)=\pi\left(w_{2}\right) .
$$

In the other direction, suppose that $\pi\left(w_{1}\right)=\pi\left(w_{2}\right)$. Then we have also that

$$
\pi\left(\sigma_{\mathbb{Z}}^{n}\left(w_{1}\right)\right)=f^{n}\left(\pi\left(w_{1}\right)\right)=f^{n}\left(\pi\left(w_{2}\right)\right)=\pi\left(\sigma_{\mathbb{Z}}^{n}\left(w_{2}\right)\right)
$$

for all $n \in \mathbb{Z}$. But this means, by definition of the transformations $T_{(a ; b)}$, that

$$
T_{\left(a_{-n} ; b_{-n}\right)}\left(a_{-n} a_{-n+1} \ldots\right)=b_{-n} b_{-n+1} \ldots,
$$

so that the sequences $w_{1}$ and $w_{2}$ are asymptotically equivalent.

\section{References}

[AP98] J. E. Anderson and I. F. Putnam. Topological invariants for substitution tilings and their associated $C^{*}$-algebras. Ergod. Th. \& Dynam. Sys., 18:509-537, 1998.

[BG00] Laurent Bartholdi and Rostislav I. Grigorchuk. On the spectrum of Hecke type operators related to some fractal groups. Proceedings of the Steklov Institute of Mathematics, 231:5-45, 2000.

[BGN03] Laurent Bartholdi, Rostislav Grigorchuk, and Volodymyr Nekrashevych. From fractal groups to fractal sets. In Peter Grabner and Wolfgang Woess, editors, Fractals in Graz 2001. Analysis - Dynamics - Geometry - Stochastics, pages 25-118. Birkhäuser Verlag, Basel, Boston, Berlin, 2003.

[BH99] Martin R. Bridson and André Haefliger. Metric spaces of non-positive curvature, volume 319 of Grundlehren der Mathematischen Wissenschaften. Springer, Berlin, 1999.

[Bow75] R. Bowen. Equilibrium states and ergodic theory of Anosov diffeomorphisms, volume 470 of Lecture Notes in Mathematics. Springer, 1975.

[BSV99] Andrew M. Brunner, Said N. Sidki, and Ana. C. Vieira. A just-nonsolvable torsion-free group defined on the binary tree. J. Algebra, 211:99-144, 1999.

[Eng77] Ryszard Engelking. General Topology, volume 60 of Monografie Matematyczne. Państwowe Wydawnictwo Naukove, Warszawa, 1977.

[Gar77] Martin Gardner. Mathematical games. Scientific American, pages 110-121, January 1977.

[GLSŻ00] Rostislav I. Grigorchuk, Peter Linnell, Thomas Schick, and Andrzej Żuk. On a question of Atiyah. C. R. Acad. Sci. Paris Sér. I Math., 331(9):663-668, 2000.

[GNS00] Rostislav I. Grigorchuk, Volodymyr V. Nekrashevich, and Vitaliu I. Sushchanskii. Automata, dynamical systems and groups. Proceedings of the Steklov Institute of Mathematics, 231:128-203, 2000.

[GPS95] Thierry Giordano, Ian F. Putnam, and Christian F. Skau. Topological orbit equivalence and $C^{*}$-crossed products. Journal fuer die reine und angewandte Mathematik, 469:51111, 1995. 
[Gri80] Rostislav I. Grigorchuk. On Burnside's problem on periodic groups. Functional Anal. Appl., 14(1):41-43, 1980.

[Gri83] Rostislav I. Grigorchuk. Milnor's problem on the growth of groups. Sov. Math., Dokl., 28:23-26, 1983.

[Gri85] Rostislav I. Grigorchuk. Degrees of growth of finitely generated groups and the theory of invariant means. Math. USSR Izv., 25(2):259-300, 1985.

[Gri98] Rostislav I. Grigorchuk. An example of a finitely presented amenable group that does not belong to the class EG. Mat. Sb., 189(1):79-100, 1998.

[Gri00] Rostislav I. Grigorchuk. Just infinite branch groups. In Aner Shalev, Marcus P. F. du Sautoy, and Dan Segal, editors, New Horizons in pro-p Groups, volume 184 of Progress in Mathematics, pages 121-179. Birkhäuser Verlag, Basel, 2000.

[GS83] Narain D. Gupta and Said N. Sidki. On the Burnside problem for periodic groups. Math. Z., 182:385-388, 1983.

[GS87] Branko Grünbaum and Goeffrey C. Shephard. Tilings and patterns. W. H. Freeman and Company, New York, 1987.

[GŻ01] Rostislav I. Grigorchuk and Andrzej Żuk. The lamplighter group as a group generated by a 2-state automaton and its spectrum. Geom. Dedicata, 87(1-3):209-244, 2001.

[Hae01] André Haefliger. Groupoids and foliations. In Groupoids in Analysis, Geometry, and Physics. AMS-IMS-SIAM joint summer research conference, University of Colorado, Boulder, CO, USA, June 20-24, 1999, volume 282 of Contemp. Math, pages 83-100. Providence, RI: A.M.S., 2001.

[Har00] Pierre de la Harpe. Topics in geometric group theory. University of Chicago Press, 2000.

[HPS92] Richard H. Herman, Ian F. Putnam, and Christian F. Skau. Ordered Bratteli diagrams, dimension groups, and topological dynamics. Intern. $\widetilde{J}$. Math., 3:827-864, 1992.

[Kel95] J. Kellendonk. Noncommutative geometry of tilings and gap labelling. Rev. Math. Phys., 7(7):1133-1180, 1995.

[Kel97] J. Kellendonk. The local structure of tilings and their integer group of coinvariants. Commun. Math. Phys., 187(1):115-157, 1997.

[Kit98] Bruce P. Kitchens. Symbolic Dynamics. Springer, Berlin etc., 1998.

[KL00] J. Kellendonk and M. V. Lawson. Tiling semigroups. J. Algebra, 224(1):140-150, 2000.

[KP00] J. Kellendonk and I. F. Putnam. Tilings, $C^{*}$-algebras, and K-theory. In Michael et al. Baake, editor, Directions in mathematical quasicrystals, volume 13 of CRM Monogr. Ser., pages 177-206. Providence, RI:AMS, American Mathematical Society., 2000.

[KPS97] J. Kaminker, I. F. Putnam, and J. Spielberg. Operator algebras and hyperbolic dynamics. In S. Doplicher, R. Longo, J.E.Roberts, and L. Zsido, editors, Operator Algebras and Quantum Field Theory. International Press, 1997.

[Kur61] Kazimierz Kuratowski. Topologie, volume II. Warszawa, 1961.

[LM95] Douglas Lind and Brian Marcus. Symbolic dynamics and coding. Cambridge University Press, 1995.

[Nek02] Volodymyr Nekrashevych. Self-similar inverse semigroups and groupoids. In Ukrainian Congress of Mathematicians: Functional Analysis, pages 176-192, 2002. 
[Nek05] Volodymyr Nekrashevych. Self-similar groups, volume 117 of Mathematical Surveys and Monographs. Amer. Math. Soc., Providence, RI, 2005.

[Pat99] Alan L. T. Paterson. Groupoids, inverse semigroups, and their operator algebras. Birkhäuser Boston Inc., Boston, MA, 1999.

[Pen84] Roger Penrose. Pentaplexity. In F. C. Holrayd and R. J. Wilson, editors, Geometrical Combinatorics, pages 55-65. Pitman, London, 1984.

[Put96] Ian F. Putnam. $C^{*}$-algebras from Smale spaces. Can. J. Math., 48:175-195, 1996.

[Ren80] Jean Renault. A groupoid approach to $C^{*}$-algebras, volume 793 of Lecture Notes in Mathematics. Springer-Verlag, Berlin, Heidelberg, New York, 1980.

[Rue78] D. Ruelle. Thermodynamic formalism. Addison Wesley, Reading, 1978.

[Sid98] Said N. Sidki. Regular Trees and their Automorphisms, volume 56 of Monografias de Matematica. IMPA, Rio de Janeiro, 1998.

[Vin00] Andrew Vince. Digit tiling of Euclidean space. In Directions in Mathematical Quasicrystals, pages 329-370. Amer. Math. Soc., Providence, RI, 2000. 